\title{
Dynamics of a simple regulatory switch.
}

\author{
Erik Boczko \\ Department of Biomedical Informatics \\ Vanderbilt University \\ Nashville, TN. 37232 \\ erik.m.boczko@vanderbilt.edu
}

\author{
Tomáš Gedeon \\ Department of Mathematical Sciences \\ Montana State University \\ Bozeman, MT 59715 \\ gedeon@math.montana.edu
}

\author{
Konstantin Mischaikow \\ Department of Mathematics \\ and \\ BioMaPS Institute \\ Hill Center-Busch Campus \\ Rutgers, The State University of New Jersey \\ 110 Frelinghusen Rd \\ Piscataway, NJ 08854-8019, USA \\ mischaik@math.rutgers.edu
}

March 29, 2007

\begin{abstract}
We consider the dynamics of a model toggle switch abstracted from the genetic interactions operative in a fungal stress response circuit. The switch transduces an external signal and propagates it forward by mediating the transport between compartments of two interacting gene products. The transport between compartments is assumed to be related to the degree of association between the interacting proteins, a fact for which there exists a wealth of biological evidence. The ubiquity and modularity of this cellular control mechanism warrants a detailed study of the dynamics entailed by various modelling assumptions. Specifically, we consider a general gate model in which both of the associating proteins are freely transportable between compartments. A more restrictive, but biologically supported model, is considered in which only one of the two proteins undergoes transport. Under the strong assumption that the disassociation of the interacting proteins is unidirectional we show that the qualitative dynamics of the two models are similar; that is they both converge to unique periodic orbits. From a biophysical perspective the assumption of unidirectional dissociation is unrealistic. We show that the same result holds for the more restrictive model when one weakens the assumption of unidirectional binding or disassociation. We speculate that this is not true for the more general model. This difference in dynamics may have important biological implications and certainly points to promising avenues of research.
\end{abstract}

\section{Introduction}

While the genomes of many species are known, and much information has been gleaned from their comparison, the promised impact of this knowledge to biology, bio-engineering, and human health cannot be fully realized until the regulation of gene expression and its dynamics are understood. Even in simple organisms the regulation of gene expression involves the interaction of many genes, signals and their products. As expected, within this complexity, and with the aid of graphical description languages, research has begun to find the promise of order. For instance, motifs common to many species have been identified [19, 20] and there is the hope that these common patterns of interaction between the genes share a common dynamic behavior. 
On the other hand many motifs that appear to be closely related to naturally occurring ones are not found among the now vast piles of experimental data. Understanding the connection between the dynamics entailed by the structure of a biological model and the frequency of this structural motif in the genomes, is perhaps the central question of systems biology.

This work represents a continuation of our earlier results on the Nitrogen Catabolite Repression (NCR) circuit in Saccharomyces cerevisiae [8]. As is explained in greater detail below, the NCR circuit contains a sub-circuit composed of two genes that functions as a nitrogen responsive toggle switch. In our earlier work we analyzed the dynamics of a model of this sub-circuit, that we shall refer to as the NCR gating switch. We draw this distinction because a purpose of the current work is to introduce and analyze the dynamics of a more general model of a toggle switch that involves an external signal, two compartments, and two periodically expressed proteins. The additional level of generality is motivated by the considerations of the previous paragraph as well as the fact that novel and ingenious regulatory mechanisms continue to be discovered at a steady pace $[25,32]$. The central regulatory mechanism involved in NCR gating switch is the control of nuclear-cytoplasmic transport, a process that can restrict how and when regulatory proteins have access to the DNA. Since NCR is studied in yeast, a eukaryote, the two compartments of the NCR gating switch model were naturally identified with the cytoplasm and the nucleus. In the more general model it is sufficient to view each compartment as a distinct localized cellular region between which transport is allowed. It is perhaps obvious but worth mentioning that in prokaryotes like E. Coli, the regulation of the nitrogen stress is accomplished by a different mechanism, and eventually when the dynamical consequences of each model are well understood these can be profitably compared.

Our continued investigation of the dynamics of this simple toggle switch and its natural generalizations is justified at least in part by the ubiquity and the modularity of its biological realization. A rudimentary literature search supports this assertion and shows that the transport gating paradigm is a rather widespread phenomena as is indicated by the list of examples in Table 1. For completeness we now describe the details of this mechanism and summarize the evidence for it.

Recent data indicate that nuclear-cytoplasmic shuttling in eukaryotes is used as a generic mechanism to control subcellular localization of effector proteins [27, 29, 31]. This layer of biological control is of interest to the clinician for whom it represents a potential target for therapeutic intervention, or the direct cause of a disease. For instance, a recent review compiled a list of ten tumor suppressor proteins for which there is solid and detailed experimental evidence of regulation via nuclear-cytoplasmic shuttling [22]. Nuclear gating of transcription factors and effector proteins is also a mechanism of great interest and importance to bioinformaticians as this layer of control cannot be detected from expression data alone.

The basic ingredients thought important for the control of nuclear-cytoplasmic shuttling are import and export sequences. Proteins have been shown to carry short amino acid sequences that act as bus passes facilitating bidirectional transport through the nuclear pore complex. In a popular model transport is regulated by hiding either the import or export sequence [27, 29, 31]. For instance, if an import sequence is hidden, the protein redistributes to the cytoplasm and vice versa. Transport sequences can be hidden by direct phosphorylation that alters a folding equilibrium, through association with other proteins, or a combination of both. Hiding or exposing sequences amounts to a toggle switch for nuclear-cytoplasmic distribution.

Nuclear-cytoplasmic transport is used as a regulatory device in the physiological stress response to nitrogen starvation in yeast. For clarity and completeness we briefly describe the essential features of the NCR process, by which yeast such as $S$. cerevisiae selectively uses good nitrogen sources (glutamine, asparagine, and ammonia in some strains) in preference to poor ones (allantoin, proline, urea). NCR-sensitive gene expression is mediated by the regulatory circuit shown in Figure $1[6,9]$. In the presence of excess nitrogen (a good nitrogen source in adequate supply) transcription of genes encoding the proteins needed to transport and degrade poor nitrogen sources occurs at only very low levels; they are, if you will, "repressed". On the other hand, when the amount of a good nitrogen source becomes limiting, or only poor nitrogen sources are available, the genes needed for their transport and catabolism are transcribed.

Depicted in Figure 1 are the four transcription factors Gln3, Gat1, Dal80 and Deh1 responsible for mediating NCR. These transcription factors are all from the GATA family of zinc-finger DNA binding proteins and they recognize and bind similar GATA containing DNA sequences to control the rate of gene expression. Since they all recognize similar or indistinguishable sequence motifs it is proposed that they act in concert at the same promoter by competition $[6,9]$. With regard to the focus of this paper an important 


\begin{tabular}{l|l|l|l}
\hline Effector Protein & Cytoplasmic Protein & Process & References \\
\hline \hline MSN2(4) & BMH2 & Carbon limitation & {$[7]$} \\
BRCA1 & BRAP2 & Tumor supressor & {$[22]$} \\
GAL80 & GAL3 & Galactose metabolism & {$[23]$} \\
FKHRL1 & 14-3-3 & Cell Proliferation & {$[4,31]$} \\
NF $\kappa$ B & I $\kappa$ B & Immune response & {$[31]$} \\
Dorsal & Cactus & Drosophila development & {$[3]$} \\
ERK & MEK & Signal transduction & {$[5,16]$} \\
Elf1 & Rb & Cell cycle & {$[14,30,27]$} \\
RSK2 & PEA-15 & Signal transduction & {$[28]$} \\
GLI-1 & SUFUH & Mammalian Development & {$[15]$} \\
\hline
\end{tabular}

Table 1: A sample of cytoplasmic gating complexes for which there is experimental evidence. With the sole exception being ERK, all of the effectors are transcription factors. ERK is a kinase that enters the nucleus and phosphorylates transcriptional regulators. In each pair when the effector disassociates from the complex it migrates to the nucleus. These protein pairs participate in a spectrum of regulatory activities from metabolite stress to tumor suppression, proliferation and cell cycle, and development.

feature of NCR is the URE2-dependent gating mechanism that keeps the circuit repressed in a good nitrogen source. This is depicted by the blunted blue arrows labeled 1 and 2 in Figure 1 . There is convincing evidence (see [9] and the references therein) to suggest that Gln3, and to a lesser extent Gat1, are sequestered in the cytoplasm in complex with Ure2, and it is hypothesized that the aggregation of the complex is driven by nitrogen state dependent phosphorylation. Under conditions of excess nitrogen, the Gln3 and Gat1 proteins are suggested to be phosphorylated and found bound to Ure2 in the cytoplasm though the precise mechanism is not understood. There is good evidence supporting the direct physical interaction of Gln3 with Ure2, there is less direct evidence of a complex with Gat1. Based on the data it is natural to model the Gln3-Ure2 interaction through the formation of a complex, $C$, that is limited to the cytoplasm. Further, we assume that there are two reactions with different rates

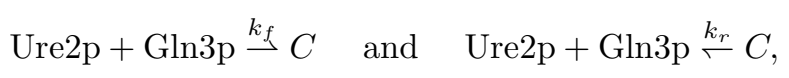

and furthermore, in the presence of excess nitrogen $k_{f}>>k_{r} \approx 0$ while in the absence of good nitrogen sources $0 \approx k_{f}<<k_{r}$.

Before delving into the specific details of the modelling we reiterate our major motivation. First, NCR is a prototypical example of a cellular stress response. Second, within the full NCR circuit there exist several important and ubiquitous control mechanisms that warrant study in their own right. Nuclear transport gating is an important example of such a control mechanism and it is perhaps the modularity of this process that makes its study relevant and ultimately useful. Finally, we remark that through analysis and generalization we can achieve both a clearer understanding of how and perhaps why the NCR circuit is a persistent feature of the yeast genome.

\subsection{Two Compartment Gating Switch}

For the sake of efficiency we begin by stating the formal mathematical model for the general two compartment gating switch and then provide a biological interpretation for each of the terms in the context of a eukaryotic 


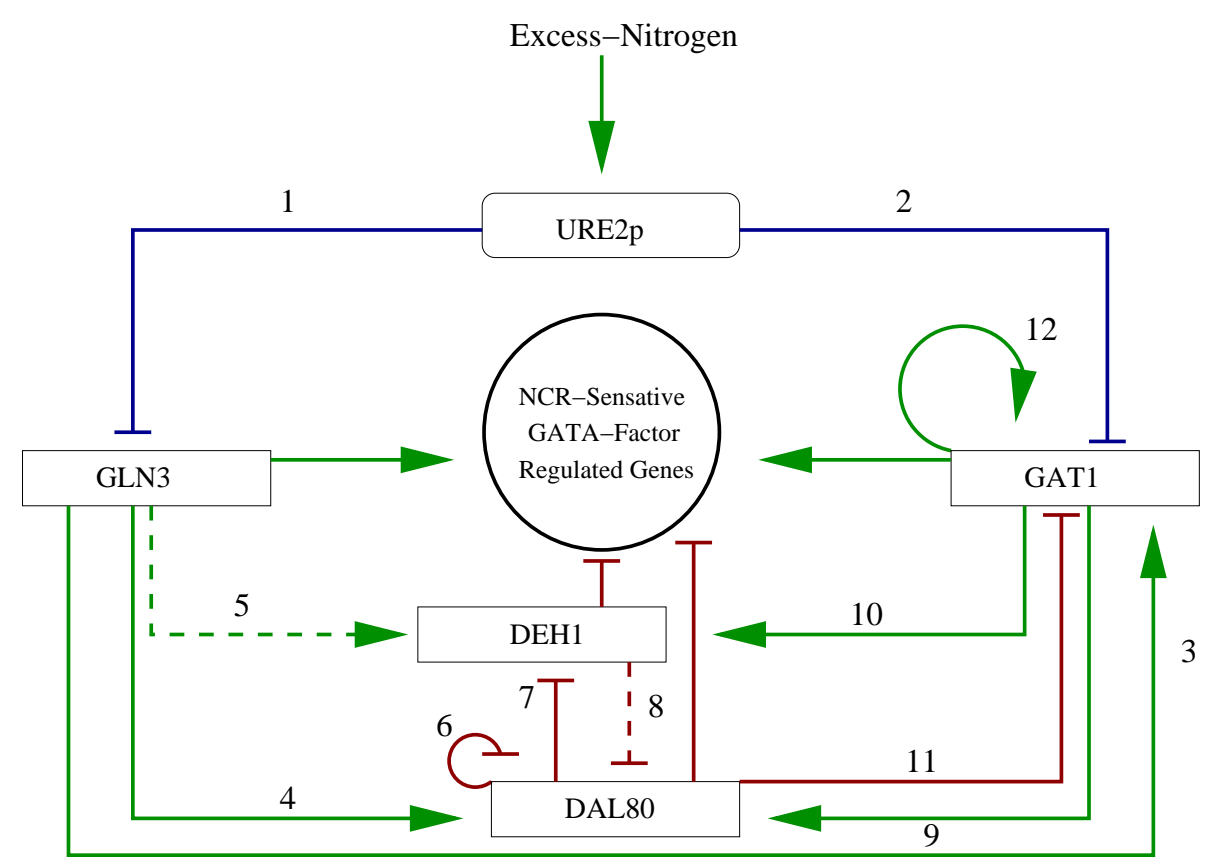

Figure 1: The NCR-circuit. Green arrows indicate up regulation, blunted red arrows represent down regulation at the level of transcription. Dashed lines indicate a weaker response. Blue lines indicate repression that is not at the transcriptional level.

cell. Consider

$$
\begin{aligned}
\dot{x} & =K_{i m p}^{0}\left(\int_{-\tau}^{0} b_{\xi}(s) \xi(t+s) d s\right)-K_{\text {exp }}^{0}(x)-\alpha x \\
\dot{X} & =h_{0}(t)-\beta X \\
\dot{\xi} & =T^{0}\left(X\left(t-\delta_{1}\right)\right)-\gamma \xi-k_{f} g(\xi, \mu)+k_{r} C+K_{e x p}^{0}\left(\int_{-\tau}^{0} b_{x}(s) x(t+s) d s\right)-K_{i m p}^{0}(\xi) \\
\dot{u} & =K_{i m p}^{1}\left(\int_{-\tau}^{0} b_{\mu}(s) \mu(t+s) d s\right)-K_{\text {exp }}^{1}(u)-\eta u \\
\dot{U} & =h_{1}(t)-\theta U \\
\dot{\mu} & =T^{1}\left(U\left(t-\delta_{2}\right)\right)-\kappa \mu-k_{f} g(\xi, \mu)+k_{r} C+K_{\text {exp }}^{1}\left(\int_{-\tau}^{0} b_{u}(s) u(t+s) d s\right)-K_{i m p}^{1}(\mu) \\
\dot{C} & =k_{f} g(\xi, \mu)-k_{r} C
\end{aligned}
$$

As is indicated in the introductory comments, two proteins lie at the heart of this regulatory switch. In the nucleus, the concentrations of these proteins are represented by $x$ and $u$. The concentrations of the same proteins in the cytoplasm are denoted by $\xi$ and $\mu$. Finally, it is assumed that these proteins are produced via translation of distinct mRNA whose concentration in the cytoplasm are indicated by $X$ and $U$, respectively.

Degradation and the volume growth of the cell decreases the concentrations of the proteins and mRNA. We assume that this happens at constant rates $\alpha, \beta, \gamma, \eta, \theta$, and $\kappa$. We assume that all these constants are strictly positive.

Since the explicit mechanisms by which the proteins are transported within and between the compartments are not assumed to be known they are modelled as follows. Distributed time delays are used to reflect the time spent in conveyance. More precisely, it is assumed that the cytoplasmic protein arriving at the nucleus at time $t$ left the cytoplasmic compartment earlier, that is at some moment in the time interval $[t, t-\tau]$ for a fixed $\tau>0$. The amount that arrives is specified by the kernel functions $b_{\xi}(s) \geq 0$ and $b_{\mu}(s) \geq 0$ with support in $[-\tau, 0]$. This gives rise to the terms $\int_{-\tau}^{0} b_{\xi}(s) \xi(t+s) d s$ and $\int_{-\tau}^{0} b_{\mu}(s) \mu(t+s) d s$. 
Similar comments apply to the transport of the proteins from the nucleus to the cytoplasm. While these terms model the amounts transported, the rates of nuclear import and export are known to be concentration dependent [24]. This is dealt with by the incorporation of nuclear import and export rate functions $K_{i m p}^{*}(\cdot)$ and $K_{e x p}^{*}(\cdot), *=0,1$.

For the moment let us assume that the proteins are constitutively expressed (we will return to this point after stating Theorem 1.3) and that the cell cycle is periodic with period $r$. Then the rate of production of the mRNA is given by periodic functions $h_{0}(t)$ and $h_{1}(t)$, respectively, with period $r$ that model the influence of the cell cycle. Notice that because of the constitutive production rate there is no apparent transcriptional time delay for these two genes.

Again, at this level of generality detailed knowledge concerning the translation process is unknown. Thus, we denote by $T^{*}(\cdot), *=0,1$ concentration dependent translation initiation rate functions, and denote by $\delta_{1}$ and $\delta_{2}$ delays that take into account the elongation and folding of the nascent protein.

The gating control mechanism is as follows. The two cytoplasmic proteins can combine, according to an association function $g(\xi, \mu)$, to form a complex whose cytoplasmic concentration is given by $C$. The rate of association of $C$ from its constituents is $k_{f}$ and rate of dissociation is $k_{r}$. As is indicated in the introductory comments, it is assumed that elements of the complex (which are necessarily in the cytoplasm) are incapable of entering the nucleus.

These comments provide the biological justification for each of the terms in (2). The precise mathematical assumptions $(\mathbf{H})$ are as follows:

1. The transport rate functions $K_{*}^{\#}, *=\exp , \mathrm{imp}$ and $\#=0,1$, are monotone bounded from above and for $s \geq 0$ satisfy $K_{*}^{\#}(s)=0$ if and only if $s=0$.

2. The kernel functions satisfy $b_{z}(s) \geq 0, b_{z}([-\tau,-\tau+\epsilon])>0$ for small $\epsilon$, and the amount of protein coming out of the transport compartment is not larger than the amount that entered the compartment. In other words,

$$
\int_{-\tau}^{T-\tau} K_{*}^{\#}(z(t)) d t \geq \int_{0}^{T} K_{*}^{\#}\left(\int_{-\tau}^{0} b_{z}(s) z(t+s) d s\right) d t
$$

for all $T \geq \tau, z=x, \xi, u, \mu, *=e x p, i m p$ and $\#=0,1$.

3. The translation initiation rate functions $T^{i}, i=0,1$, are positive for $s>0$.

4. The partial derivatives of the rate function $\frac{\partial g}{\partial \xi}>0$ and $\frac{\partial g}{\partial \mu}>0$ are positive.

5. The right-hand side of system (2) needs to be at least $C^{1}$.

Observe that (2) is an $r$-periodic system of delay-differential equations, where $r$ is the minimal period of $h_{0}(t)$ and $h_{1}(t)$. Since the quantities $x, X, \xi, u, U$ and $\mu$ enter the right hand side of (2) delayed, in order to compute the right hand side at time $t$ we need to know the values of $x, \xi, u$ and $\mu$ on the entire interval $[t-\tau, t]$. Similarly $X$ must be known for $\left[t-\delta_{1}, t\right]$ and $U$ on the interval $\left[t-\delta_{2}, t\right]$. The variable $C$ needs to be known only at time $t$. In order to simplify the notation we assume that $\tau>\delta_{1}$ and $\tau>\delta_{2}$. This is not a significant assumption since in the opposite case we can take the length of the delay interval to be the maximum of $\tau, \delta_{1}$ and $\delta_{2}$.

To introduce the phase space for this dynamical system, let $C\left([-\tau, 0], \mathbf{R}^{n}\right)$ be a Banach space of continuous functions on $[-\tau, 0]$ with range $\mathbf{R}^{n}$. If we set

$$
y_{t}(\theta):=y(t+\theta)-\tau \leq \theta \leq 0,
$$

then $y_{t}$ belongs to the space $C\left([-\tau, 0], \mathbf{R}^{n}\right)$. The phase space for $(2)$ is

$$
\left(x_{t}(\theta), X_{t}(\theta), \xi_{t}(\theta), u_{t}(\theta), \mu_{t}(\theta), U_{t}(\theta), C\right) \in \mathcal{D}:=C^{1}\left([-\tau, 0], \mathbf{R}^{6}\right) \times \mathbf{R}
$$

System (2) defines a semi-flow $\Psi\left(t, t_{0}, w\right):[0, \infty) \times[0, r] \times \mathcal{D} \rightarrow \mathcal{D}$ which is fully characterized by a time $r$ map $H: \mathcal{D} \rightarrow \mathcal{D}$

$$
H(w):=\Psi(r, 0, w)
$$


Definition 1.1. [11, Chapter 3.6] Suppose $X$ is a complete metric space, $\mathbf{R}^{+}=[0, \infty)$, and $u: \mathbf{R}^{+} \times \mathbf{R} \times X \rightarrow$ $X$ is a given mapping. Define $U(t, \sigma): X \rightarrow X$ for $t \in \mathbf{R}^{+}, \sigma \in \mathbf{R}$ by $U(t, \sigma) x=u(t, \sigma, x)$. An $\omega$-periodic process is a function $u$ satisfying following properties:

1. $u$ is continuous;

2. $U(0, \sigma)=I$, the identity;

3. $U(t, \sigma+s) U(s, \sigma)=U(s+t, \sigma)$;

4. there is $\omega>0$ such that $U(t, \sigma+\omega)=U(t, \sigma)$ for all $t \in \mathbf{R}^{+}$and $\sigma \in \mathbf{R}$.

Observe, that the map $H$, defined above, generates an $r$-periodic process.

We will use the following result about periodic processes.

Theorem 1.2. [11, Theorem 3.6.3] If $X$ is a Banach space, the $\omega$-periodic process is completely continuous and is point dissipative, then there is a connected global attractor $A_{\sigma}$ for each $\sigma$. Furthermore, there exists an $\omega$-periodic trajectory of the process.

Let $\mathcal{D}^{+}$be the nonnegative cone of $\mathcal{D}$, i.e. the space

$$
\begin{aligned}
\mathcal{D}^{+}:= & \left\{\left(x_{t}(\theta), X_{t}(\theta) \xi_{t}(\theta), u_{t}(\theta), \mu_{t}(\theta), U_{t}(\theta), C\right) \in \mathcal{D} \mid x_{t}(\theta) \geq 0, X_{t}(\theta) \geq 0,\right. \\
& \left.\xi_{t}(\theta) \geq 0, u(\theta) \geq 0, \mu(\theta) \geq 0, U_{t}(\theta) \geq 0, C \geq 0 \text { for all } \theta \in[-\tau, 0]\right\} .
\end{aligned}
$$

It is easy to verify that if an initial condition of (2) has each of its components non-negative, then this property will remain unchanged along the entire solution for all $t \geq 0$. Therefore, we can restrict our phase space to the biologically relevant positive cone $\mathcal{D}^{+}$of phase space $\mathcal{D}$. Standard results $[11,26]$ guarantee existence and uniqueness of solutions of (2).

Recall that the gating is controlled by the formation of the complex $C$ and as is indicated in (1) the constants $k_{f}$ and $k_{r}$ determine whether the proteins are free or sequestered in the complex $C$. The following theorem provides a precise description of the asymptotic dynamics in the case in which either $k_{f}=0$ or $k_{r}=0$.

Theorem 1.3. Consider (2) and assume the hypothesis $(\mathbf{H})$ are satisfied.

a. If $k_{f}=0$ and $r \geq 2 \tau$, then there is a unique $r$ periodic solution $\Gamma\left(t, t_{0}\right)$ which is a global attractor for $w \in \mathcal{D}^{+}$.

b. If $k_{r}=0$ and $r \geq 3 \tau$ then there is a unique $r$ periodic solution $\Gamma\left(t, t_{0}\right)$ which is a global attractor for $w \in \mathcal{D}^{+}$.

Obviously, this theorem implies that if the cell cycle period is sufficiently long as compared to the transport time in the cell, then this two compartment gating switch leads to unique period oscillations of the protein and mRNA levels with the same period as that of the cell cycle. An important corollary of this is that we can weaken one of the biological assumptions made in constructing the model. In the original biological description of the model it was assumed that the proteins are constitutively expressed. This led to the periodic production of the associated mRNA. However, this theorem implies that the output from a two compartment gating switch is periodic with period of the cell cycle, and hence we can now generalize the assumption and allow the proteins themselves to be controlled by an upstream two compartment gating switch. Though, in and of itself, this is a simple observation, it does suggest that the long term goal of being able to understand gene expression and control on the level of the organism can benefit from the analysis of sub-circuit dynamics. We will return to this point in the concluding remarks of the paper.

\subsection{NCR Gating Switch}

The weakness of Theorem 1.3 lies in the fact that it requires the assumption that either $k_{f}=0$ or $k_{r}=0$. In any true chemical reaction, the reaction rates are always positive, though they may be exceedingly small. We do not know whether, in the complete generality of the two compartment gating switch, the conclusion is stable with respect to perturbations of the reaction rates. Thus, to strengthen the result we turn our attention once again to the NCR circuit.

A specific identification of NCR with (2) can be made as follows: 
- let $\xi$ and $\mu$ represent cytoplasmic Gln3p and Ure2p, respectively,

- let $X$ and $U$ represent GLN3 and URE2 mRNA, respectively,

- let $x$ and $u$ represent nuclear Gln3p and Ure2p, respectively.

However, there is strong evidence (see [9] and the references therein) that Ure2p does not enter the nucleus and stays in the cytoplasm. Therefore,

$$
u \equiv 0
$$

and (2) reduces to

$$
\begin{aligned}
\dot{x} & =K_{i m p}\left(\int_{-\tau}^{0} b_{\xi}(s) \xi(t+s) d s\right)-K_{\exp }(x)-\alpha x \\
\dot{X} & =h_{0}(t)-\beta X \\
\dot{\xi} & =T^{0}\left(X\left(t-\delta_{1}\right)\right)-\gamma \xi-k_{f} g(\xi, \mu)+k_{r} C+K_{\exp }\left(\int_{-\tau}^{0} b_{x}(s) x(t+s) d s\right)-K_{i m p}(\xi) \\
\dot{U} & =h_{1}(t)-\theta U \\
\dot{\mu} & =T^{1}\left(U\left(t-\delta_{2}\right)\right)-\kappa \mu-k_{f} g(\xi, \mu)+k_{r} C \\
\dot{C} & =k_{f} g(\xi, \mu)-k_{r} C
\end{aligned}
$$

Notice that we have dropped the unnecessary superscripts on $K_{i m p}$ and $K_{\exp }$.

The phase space for (3) is

$$
\left(x_{t}(\theta), X_{t}(\theta), \xi_{t}(\theta), U_{t}(\theta), \mu, C\right) \in \mathcal{D}^{\mathrm{NCR}}:=C^{1}\left([-\tau, 0], \mathbf{R}^{4}\right) \times \mathbf{R}^{2} .
$$

Notice that the variable $\mu$ only needs to be known at the present time $t$.

Let $\mathcal{D}^{\mathrm{NCR}+}$ denote the positive cone in $\mathcal{D}^{\mathrm{NCR}}$.

For this system we have the following result that allows for more realistic assumptions on the reaction rates for the gating complex.

Theorem 1.4. Consider (3) and assume hypothesis $(\mathbf{H})$ holds.

a. There exists $\epsilon_{f}>0$, such that if $0 \leq k_{f}<\epsilon_{f}$ and $r \geq 2 \tau$, then there exists a unique $r$ periodic solution $\Gamma\left(t, t_{0}\right)$ which is a global attractor for $w \in \mathcal{D}^{\mathrm{NCR}+}$.

b. There exists $\epsilon_{r}>0$, such that if $0 \leq k_{r}<\epsilon_{r}$ and $r \geq 3 \tau$, then there exists a unique $r$ periodic solution $\Gamma\left(t, t_{0}\right)$ which is a global attractor for $w \in \mathcal{D}^{\mathrm{NCR}+}$.

\subsection{Outline of Paper}

This paper is organized as follows. Observe that in the systems (2) and (3) the $X$ and $U$ equations can be solved independently from the others. As $\tau \rightarrow \infty$ the solutions of (2) and (3) approach solutions of corresponding asymptotic systems which are described in section 2 . These asymptotic systems are studied in section 3 under the assumption $k_{f}=0$. There are three essential points that are discussed: global estimates (Section 3.3), monotonicity (Section 3.4), and the introduction of a Lyapunov function (Section 3.5). Together these allow us to prove Theorem 1.3.a. In Section 4, these ideas are used again to prove the perturbation result Theorem 1.4.a. In Section 5 we prove the corresponding results for $k_{r}=0$. Since the arguments are similar to the $k_{f}=0$ case, we only highlight the differences. In particular, the monotonicity results require a different cone and a modified Lyapunov function is required. Finally, in Section 6 we conclude by speculating on the implications of the differences in the structure of the NCR gate versus the general gate. 


\section{Asymptotic system}

Since our theorems concern the existence of a globally attracting periodic orbit, it is reasonable to try to reduce (2) by considering its asymptotic dynamics. Observe that one can solve explicitly for $X(t)$ and $U(t)$ :

$$
\begin{aligned}
& X(t)=X(0) e^{-\beta t}+e^{-\beta t} \int_{0}^{t} e^{\beta s} h_{0}(s) d s \\
& U(t)=U(0) e^{-\theta t}+e^{-\theta t} \int_{0}^{t} e^{\theta s} h_{1}(s) d s
\end{aligned}
$$

Since $h_{0}$ and $h_{1}$ are $r$-periodic, $X(t) \rightarrow \bar{X}(t)$ and $U(t) \rightarrow \bar{U}(t)$ as $t \rightarrow \infty$, where both $\bar{X}(t)$ and $\bar{U}(t)$ are $r$-periodic functions of time.

Therefore, (2) is asymptotic in time to the system

$$
\begin{array}{rlr}
\dot{x}= & K_{\text {imp }}^{0}\left(\int_{-\tau}^{0} b_{\xi}(s) \xi(t+s) d s\right)-K_{\text {exp }}^{0}(x)-\alpha x \\
\dot{\xi}= & T^{0}\left(\bar{X}\left(t-\delta_{1}\right)\right)-\gamma \xi-k_{f} g(\xi, \mu)+k_{r} C \\
& \quad+K_{\text {exp }}^{0}\left(\int_{-\tau}^{0} b_{x}(s) x(t+s) d s\right)-K_{i m p}^{0}(\xi) \\
\dot{u}= & K_{i m p}^{1}\left(\int_{-\tau}^{0} b_{\mu}(s) \mu(t+s) d s\right)-K_{\text {exp }}^{1}(u)-\eta u \\
\dot{\mu}= & T^{1}\left(\bar{U}\left(t-\delta_{2}\right)\right)-\kappa \mu-k_{f} g(\xi, \mu)+k_{r} C \\
& \quad+K_{\text {exp }}^{1}\left(\int_{-\tau}^{0} b_{u}(s) u(t+s) d s\right)-K_{i m p}^{1}(\mu) \\
\dot{C}= & k_{f} g(\xi, \mu)-k_{r} C
\end{array}
$$

As is indicated in the introduction, $(2)$ generates a semi-flow $\Psi\left(t, t_{0}, w\right):[0, \infty) \times[0, r] \times \mathcal{D} \rightarrow \mathcal{D}$. Let $\mathcal{C}:=C^{1}\left([-\tau, 0], \mathbf{R}^{4}\right) \times \mathbf{R}$ be a subset of $\mathcal{D}$. We view the functions $X(t)$ and $U(t)$ as a non-autonomous input to the equations restricted to $\mathcal{C} \subset \mathcal{D}$ and let $\Phi\left(t, t_{0}, w\right)$ denote the corresponding (non-autonomous) semi-flow defined by $(2)$ on the space $\mathcal{C}$. The corresponding equations are those of $(5)$ where the periodic functions $\bar{X}$ and $\bar{U}$ are replaced by functions $X(t)$ and $U(t)$ obeying the differential equations in (2). Let

$$
S_{m}(w):=\Phi(m r,(m-1) r, w), \quad \text { for integers } m \geq 1,
$$

$S_{m}: \mathcal{C} \rightarrow \mathcal{C}$ and define maps $T_{n}$ by

$$
T_{n}=S_{n-1} \circ S_{n-2} \circ \ldots \circ S_{1} \circ S_{0}: \mathcal{C} \rightarrow \mathcal{C}, n \geq 1
$$

with $T_{0}=I$, where $I: \mathcal{C} \rightarrow \mathcal{C}$ is the identity mapping on $\mathcal{C}$.

Definition 2.1 ([35]). Let $T_{n}: X \rightarrow X$ be a discrete dynamical process defined in (6) and $S: X \rightarrow X$ a continuous map. The collection $\left\{T_{n}\right\}_{n \geq 0}$ is called an asymptotically autonomous process with limit discrete semi-flow $\left\{S^{n}\right\}_{n \geq 0}$, generated by the iterates of $S$, if

$$
S_{m_{j}}\left(x_{j}\right) \rightarrow S(x), j \rightarrow \infty
$$

for any two sequences $m_{j} \rightarrow \infty, x_{j} \rightarrow x$, as $j \rightarrow \infty$, with $x, x_{j} \in X$.

Observe, that if we set $\varphi\left(t, t_{0}, w_{0}\right): \mathbf{R} \times[0, r] \times \mathcal{C} \rightarrow \mathcal{C}$ to be the semi-flow generated by $(5)$ and

$$
S(w):=\varphi(r, 0, w)
$$

then $\left\{T_{n}\right\}_{n \geq 0}$ is an asymptotically autonomous process with limit discrete semi-flow $S^{n}(w)$ generated by iterations of the map $S(w)$. 


\subsection{Asymptotic system for the NCR gate}

The key difference between the NCR gate equations (3) and the general system (2) is the lack of the $u$ equation in the former system. This means that the asymptotic system for (5) takes the form

$$
\begin{aligned}
\dot{x}= & K_{i m p}\left(\int_{-\tau}^{0} b_{\xi}(s) \xi(t+s) d s\right)-K_{\exp }(x)-\alpha x \\
\dot{\xi}= & T^{0}\left(\bar{X}\left(t-\delta_{1}\right)\right)-\gamma \xi-k_{f} g(\xi, \mu)+k_{r} C \\
& \quad+K_{\exp }\left(\int_{-\tau}^{0} b_{x}(s) x(t+s) d s\right)-K_{i m p}(\xi) \\
\dot{\mu}= & T^{1}\left(\bar{U}\left(t-\delta_{2}\right)\right)-\kappa \mu-k_{f} g(\xi, \mu)+k_{r} C \\
\dot{C}= & k_{f} g(\xi, \mu)-k_{r} C,
\end{aligned}
$$

where again we dropped the unnecessary superscripts. Recall that for the NCR gate, $\mathcal{D}^{N C R}:=C^{1}\left([-\tau, 0], \mathbf{R}^{4}\right) \times$ $\mathbf{R}^{2}$. Analogous to the general gate, let $\mathcal{C}^{N C R}:=C^{1}\left([-\tau, 0], \mathbf{R}^{2}\right) \times \mathbf{R}^{2}$ be a subspace of $\mathcal{D}^{N C R}$. Equations (3) define the flow $\Psi^{N C R}\left(t, t_{0}, w\right)$ on $\mathcal{D}^{N C R}$ and let $\Phi^{N C R}\left(t, t_{0}, w\right)$ be a (non-autonomous) semi-flow, defined by (3) on the space $\mathcal{C}^{N C R}$. The corresponding equations are those of (7) where the periodic functions $\bar{X}$ and $\bar{U}$ are replaced by functions $X(t)$ and $U(t)$ obeying differential equations in (3). We define in an analogous way to the general case the asymptotically autonomous process $\left\{T_{n}^{N C R}\right\}_{n \geq 0}$ with limit discrete semi-flow $\left(S^{N C R}\right)^{n}(w)$ generated by iterations of the map $S^{N C R}(w)$ where $S^{N C R}(w):=\varphi^{N C R}(r, 0, w)$ and $\varphi^{N C R}\left(t, t_{0}, w_{0}\right): \mathbf{R} \times[0, r] \times \mathcal{C}^{N C R} \rightarrow \mathcal{C}^{N C R}$ is the semi-flow generated by (7).

We now proceed to analyze the dynamics of the asymptotic systems (5) and (7) in cases when $k_{f}=0$ and when $k_{r}=0$. We first deal with the case $k_{f}=0$.

\section{The open gate: $k_{f}=0$}

If $0=k_{f}<k_{r}$, then (1) implies that the complex $C$ is disassociating into the two proteins, which can in principle leave the cytoplasmic compartment. Since the analysis of the general gate (5) and the NCR gate (7) overlap, we proceed with their analysis in parallel tracks.

\subsection{The general gate}

Observe that with $k_{f}=0$, (5) reduces to

$$
\begin{aligned}
\dot{x}= & K_{i m p}^{0}\left(\int_{-\tau}^{0} b_{\xi}(s) \xi(t+s) d s\right)-K_{\text {exp }}^{0}(x)-\alpha x \\
\dot{\xi}= & T^{0}\left(\bar{X}\left(t-\delta_{1}\right)\right)-\gamma \xi+k_{r} C \\
& \quad+K_{\text {exp }}^{0}\left(\int_{-\tau}^{0} b_{x}(s) x(t+s) d s\right)-K_{i m p}^{0}(\xi) \\
\dot{u}= & K_{i m p}^{1}\left(\int_{-\tau}^{0} b_{\mu}(s) \mu(t+s) d s\right)-K_{\text {exp }}^{1}(u)-\eta u \\
\dot{\mu}= & T^{1}\left(\bar{U}\left(t-\delta_{2}\right)\right)-\kappa \mu+k_{r} C \\
& \quad+K_{\exp }^{1}\left(\int_{-\tau}^{0} b_{u}(s) u(t+s) d s\right)-K_{i m p}^{1}(\mu) \\
\dot{C}= & -k_{r} C
\end{aligned}
$$

We denote by $K^{G}$ the period $r$ map for the semi-flow generated by (8). Clearly, since $k_{r}>0, C$ converges exponentially to zero. Let $\mathcal{B}:=C^{1}\left([-\tau, 0], \mathbf{R}^{4}\right)$ and denote its positive cone by $\mathcal{B}^{+}$. The set

$$
\mathcal{B} \times\{0\} \subset \mathcal{B} \times \mathbf{R}
$$


is an invariant, exponentially attracting manifold under the map $K^{G}$.

Let $H^{G}$ be the restriction of the map $K^{G}$ to the manifold $\mathcal{B} \times\{0\} \cong \mathcal{B}$. Then $H^{G}(w)$ is the period $r$ map of the system

$$
\begin{aligned}
\dot{x} & =K_{\text {imp }}^{0}\left(\int_{-\tau}^{0} b_{\xi}(z) \xi(t+z) d z\right)-K_{\text {exp }}^{0}(x)-\alpha x \\
\dot{\xi} & =T^{0}\left(\bar{X}\left(t-\delta_{1}\right)\right)-\gamma \xi+K_{\text {exp }}^{0}\left(\int_{-\tau}^{0} b_{x}(z) x(t+z) d z\right)-K_{i m p}^{0}(\xi), \\
\dot{u} & =K_{i m p}^{1}\left(\int_{-\tau}^{0} b_{\mu}(s) \mu(t+s) d s\right)-K_{\text {exp }}^{1}(u)-\eta u \\
\dot{\mu} & =T^{1}\left(\bar{U}\left(t-\delta_{2}\right)\right)-\kappa \mu+K_{\text {exp }}^{1}\left(\int_{-\tau}^{0} b_{u}(s) u(t+s) d s\right)-K_{\text {imp }}^{1}(\mu)
\end{aligned}
$$

Note that the first two and the last two equations decouple and thus the map $K^{G}$ can be decomposed as

$$
K^{G}:=\left(H^{G}, E^{G}\right):=\left(F_{x}, F_{u}, E^{G}\right)
$$

where $F_{x}$ is a period $r$-map of the flow generated by the first two equations, $F_{u}$ the period $r$ map generated by the last two equations in (9), and $E^{G}$ is a period $r$ map generated by the last equation of (8).

\subsection{The NCR gate}

For the NCR gate (9) reduces further to

$$
\begin{aligned}
\dot{x} & =K_{i m p}\left(\int_{-\tau}^{0} b_{\xi}(z) \xi(t+z) d z\right)-K_{\exp }(x)-\alpha x \\
\dot{\xi} & =T^{0}\left(\bar{X}\left(t-\delta_{1}\right)\right)-\gamma \xi+K_{\exp }\left(\int_{-\tau}^{0} b_{x}(z) x(t+z) d z\right)-K_{i m p}(\xi), \\
\dot{\mu} & =T^{1}\left(\bar{U}\left(t-\delta_{2}\right)\right)-\kappa \mu
\end{aligned}
$$

Since $\bar{U}(t)$ is an $r$-periodic function, for all initial conditions the solutions of

$$
\dot{\mu}=T^{1}\left(\bar{U}\left(t-\delta_{2}\right)\right)-\kappa \mu
$$

converge to a unique $r$-periodic function $\bar{\mu}(t)$.

Let $\mathcal{E}:=C^{1}\left([-\tau, 0], \mathbf{R}^{2}\right)$ and denote its positive cone by $\mathcal{E}^{+}$. Let $K_{0}$ denote the map $S^{N C R}$ for the semi-flow generated by $(7)$ when $k_{f}=0$.

Lemma 3.1. The set

$$
\mathcal{E} \times \bar{\mu}(0) \times\{0\} \subset \mathcal{E} \times \mathbf{R}^{2},
$$

is an invariant, exponentially attracting manifold under the map $K_{0}$.

Proof. The map $K_{0}$ is the period map for the flow $(7)$ when $k_{f}=0$. The last two equations in (7) when $k_{f}=0$ have the form

$$
\begin{aligned}
\dot{\mu} & =T^{1}\left(\bar{U}\left(t-\delta_{2}\right)\right)-\kappa \mu+k_{r} C \\
\dot{C} & =-k_{r} C .
\end{aligned}
$$

It follows from a direct computation that all the trajectories approach $\mathcal{E} \times \bar{\mu}(0) \times\{0\}$ exponentially with rates $k_{r}$ and $\kappa$. 
Let $F_{0}$ be the restriction of the map $K_{0}$ to the manifold $\mathcal{E} \times \bar{\mu}(0) \times\{0\}$. Then $F_{0}(w):=T_{0}(r, 0, w)$ is a period $r$ map, where $T_{0}\left(t, t_{0}, w_{0}\right)$ is the solution at time $t$ of the system

$$
\begin{aligned}
& \dot{x}=K_{i m p}\left(\int_{-\tau}^{0} b_{\xi}(z) \xi(t+z) d z\right)-K_{\exp }(x)-\alpha x \\
& \dot{\xi}=T\left(\bar{X}\left(t-\delta_{1}\right)\right)-\gamma \xi+K_{\exp }\left(\int_{-\tau}^{0} b_{x}(z) x(t+z) d z\right)-K_{i m p}(\xi) .
\end{aligned}
$$

Note that the map $K_{0}$ can be decomposed as

$$
K_{0}=\left(F_{0}, E_{0}\right)
$$

where $E_{0}$ is a period $r$-map of the flow generated by (11).

Remark 3.2. Comparing (10) and (13) we see that the maps $K^{G}$ and $K_{0}$ are composed of trivially convergent maps $E^{G}$ and $E_{0}$ and the maps $F_{x}, F_{u}$ and $F_{0}$. The latter three maps are all period $r$ maps of a system of the form (12). In fact, the maps $F_{x}$ and $F_{0}$ are identical. The reason for the distinct notation is that we will make a perturbation argument for the NCR circuit at which point it is convenient to denote the perturbation of $F_{0}$ by $F_{\epsilon}$. In conclusion, to analyze both $K^{G}$ and $K_{0}$ we need to understand the period $r$ map of the system (12).

\subsection{Point dissipativity and complete continuity}

To obtain the desired convergence results we need to prove that the system exhibits sufficient asymptotic regularity and compactness. We begin by recalling some standard terminology and results for delay equations from Hale [11] and Hale and Verduyn-Lunel [12].

Definition 3.3. Let $X$ and $Y$ be two metric spaces. A continuous map $A: X \rightarrow Y$ is bounded (completely continuous) if for any closed bounded set $B$, the image $A(B)$ is bounded (the closure of $A(B)$ is compact). If $A(\lambda): X \rightarrow Y$ depends on a parameter in a metric space $\Lambda$, then $A(\lambda)$ is said to be bounded uniformly on compact sets of $\Lambda$ if, for any compact set $\Lambda_{0} \subset \Lambda$ and any bounded set $X_{0} \subset X$, there is a bounded set $Y_{0} \subset Y$ such that $A(\lambda) X_{0} \subset Y_{0}$ for all $\lambda \in \Lambda_{0}$.

Definition 3.4. Consider a metric space $X$ and continuous map $A: X \rightarrow X$. If $A$ maps any bounded set to a subset of a compact set, then it is completely continuous. It is asymptotically smooth if, for any bounded set $B$ for which $A B \subset B$, there exists a compact set $K$ such that $\operatorname{dist}\left(A^{n} B, K\right) \rightarrow 0$ as $n \rightarrow \infty$. Finally, it is point dissipative, if there exists a bounded set $B$ (independent on $x$ ) such that every trajectory $A^{n}(x)$ enters $B$ and stays there.

Theorem 3.5. [11, Corollary 3.6.2] Consider $x^{\prime}=f\left(t, x_{t}\right)$ where $f: \mathbf{R} \times C\left([-\tau, 0], \mathbf{R}^{n}\right) \rightarrow \mathbf{R}^{n}$. If $f$ is a bounded continuous map and the solution map $A\left(t_{0}, t, \cdot\right): C\left([-\tau, 0], \mathbf{R}^{n}\right) \rightarrow C\left([-\tau, 0], \mathbf{R}^{n}\right)$ is bounded uniformly on compact sets of $\left[t_{0}, \infty\right)$, then $A\left(t_{0}, t, \cdot\right)$ is completely continuous for all $t \geq t_{0}+\tau$.

Invariant regions provide a means to verify the hypothesis of Theorem 3.5. Since the theory of monotone dynamical systems (see H. Smith [26]) plays an essential role in the proofs of our principle results, we use this language to describe the appropriate invariant regions.

A closed convex cone $D \subset C\left([-\tau, 0], \mathbf{R}^{n}\right)$ is an order cone if $D \cap(-D)=\{0\}$. Given an order cone $D \subset C([-\tau, 0]$, define an order on $C([-\tau, 0]$ by writing $\varphi \succeq \psi$ if, and only if, $\varphi-\psi \in D$. It is standard to write $\varphi \succ \psi$ if $\varphi \succeq \psi$ and $\varphi \neq \psi$. If the cone $D$ has nonempty interior, then $\varphi \succ \succ \psi$ if, and only if, $\varphi-\psi \in \operatorname{int} D$. To prove the desired dissipativity and complete continuity results we make use of two different orders. In case $k_{f}=0$ we will use the following order. Let $D$ be the cone of nonnegative functions in $C\left([-\tau, 0]\right.$. Define $\varphi \succeq \psi$ if, and only if, $\varphi_{i}-\psi_{i} \geq 0$, and $\varphi \succ \succ \psi$ if, and only if, $\varphi_{i}-\psi_{i}>0$, for all $i$.

For a constant $a \in \mathbf{R}^{n}$ let $\hat{a}$ denote the obvious embedding of $a$ into $C\left([-\tau, 0], \mathbf{R}^{n}\right)$. Similarly, $[\hat{0}, \hat{k}]$ is taken to be an interval in the positive cone $C^{+}$of the set $C\left([-\tau, 0], \mathbf{R}^{n}\right)$; that is, the set of all $\varphi \in C\left([-\tau, 0], \mathbf{R}^{n}\right)$ such that $0 \leq \varphi_{i} \leq \hat{k}_{i}$. 
Lemma 3.6. [26, Theorem 5.2.1 and Remark 5.2.1] Consider a system $w^{\prime}=f\left(w_{t}\right)$ on $C=C\left([-\tau, 0], \mathbf{R}^{n}\right)$ where $f$ is $C^{1}$. The interval $[\hat{0}, \hat{k}]$, for some $k \in \mathbf{R}^{n}$, is positively invariant provided that whenever $w \in[\hat{0}, \hat{k}]$ and $w_{i}(0)=0\left(w_{i}(0)=k_{i}\right)$ for some $i$ then $f_{i}(t, w) \geq 0\left(f_{i}(t, w) \leq 0\right)$ for all $t$.

Returning to the gating problem, for the NCR model we will make use of the period maps generated by the system (7). Define $K_{\epsilon}(w):=T_{\epsilon}(r, 0, w)$ to be a period map on $\mathcal{C}^{N C R}$ of the semiflow (7) with $k_{f}=\epsilon<<1$.

Lemma 3.7 provides the basic dissipation estimate not only for the maps $F_{0}, F_{x}$ and $F_{u}$ but also for the perturbations $K_{\epsilon}$ of the map $K_{0}=\left(F_{0}, E_{0}\right)$.

Lemma 3.7. a. There exists an $\epsilon_{0}>0$ such that for all $\epsilon \in\left[0, \epsilon_{0}\right)$ the map $K_{\epsilon}$, is point dissipative and completely continuous.

b. $F_{0}$ is point dissipative and completely continuous.

c. $H^{G}:=\left(F_{x}, F_{u}\right)$ is point dissipative and completely continuous.

Proof. (a). We need to verify the assumptions of Theorem 3.5 for system (7). The right hand side $f^{1}$ of (7) is clearly bounded and continuous so we need to show that the solution map is bounded uniformly on compact subsets of $\left[t_{0}, \infty\right)$. Set

$$
\begin{aligned}
U_{0} & :=\max _{u} K_{i m p}(u), & U_{1} & :=\max _{t \in[0, r]} T^{0}(\bar{X}(s)) \\
U_{2} & :=\max _{u} K_{\exp }(u), & U_{3} & :=\max _{t \in[0, r]} T^{1}(\bar{U}(s))
\end{aligned}
$$

Define

$$
\begin{array}{cl}
R_{x}:=\frac{U_{0}}{\alpha}, & R_{\xi}:=\frac{U_{1}+U_{2}}{\gamma}, \\
R_{C}:=\min \left\{k_{f} \frac{g\left(R_{\xi}, R_{x}\right)}{k_{r}}, \frac{K_{i m p}\left(R_{\xi}\right)}{k_{r}}\right\}, & R_{\mu}:=\frac{U_{3}+k_{r} R_{C}}{\kappa},
\end{array}
$$

and

$$
R:=\left(R_{x}, R_{\xi}\right), \quad U:=\left(R_{\mu}, R_{C}\right) .
$$

To verify the conditions of Lemma 3.6 for an order interval $Q:=[\hat{0}, \hat{R}] \times[\hat{0}, \hat{U}]$ consider an initial condition $w_{0}=\left(x_{0}, \xi_{0}, \mu_{0}, C_{0}\right)$. If $x_{0}(0)=0$ and $w_{0} \in Q$, then $f_{1}^{1}\left(w_{0}\right)=K_{i m p}\left(\xi_{0}(-\tau)\right) \geq 0$. On the other hand, if $x_{0}(0)=R_{x}$ and $w_{0} \in Q$, then

$$
\begin{aligned}
f_{1}^{1}\left(w_{0}\right) & =K_{i m p}\left(\xi_{0}(-\tau)\right)-K_{\exp }\left(x_{0}(0)\right)-a x_{0}(0) \\
& \leq U_{0}-\alpha R_{x}-K_{\exp }\left(R_{x}\right) \\
& \leq-K_{\exp }\left(R_{x}\right) \\
& <0
\end{aligned}
$$

For the second coordinate we let $\xi_{0}(0)=0$ and $w_{0} \in Q$. Then, for all sufficiently small $k_{f}=\epsilon$,

$$
f_{2}^{1}\left(w_{0}\right)=T^{0}\left(\bar{X}\left(t-\delta_{1}\right)\right)+K_{e x p}\left(x_{0}(-\tau)\right)-k_{f} g(\xi, \mu)+k_{r} C>0 .
$$

If $\xi_{0}(0)=R_{\xi}$ and $w_{0} \in Q$, then

$$
\begin{aligned}
f_{2}^{1}\left(w_{0}\right) & =T^{0}\left(\bar{X}\left(t-\delta_{1}\right)\right)-\gamma \xi_{0}(0)-K_{i m p}\left(\xi_{0}(-\tau)\right)+K_{e x p}\left(x_{0}(-\tau)\right)+k_{r} C-k_{f} g(\xi, \mu) \\
& \leq U_{1}-\gamma R_{\xi}-K_{i m p}\left(R_{\xi}\right)+K_{e x p}\left(x_{0}(0)\right)+k_{r} R_{C} \\
& <U_{1}+U_{2}-\gamma R_{\xi} \\
& \leq 0 .
\end{aligned}
$$

For the third coordinate let $\mu_{0}=0$ and $w_{0} \in Q$. Then

$$
f_{3}^{1}\left(w_{0}\right)=T^{1}\left(\bar{U}\left(t-\delta_{2}\right)\right)-k_{f} g(\xi, \mu)+k_{r} C>0
$$


for all sufficiently small $k_{f}=\epsilon$. If $\mu_{0}=R_{\mu}$ and $w_{0} \in Q$ we have

$$
\begin{aligned}
f_{3}^{1}\left(w_{0}\right) & =T^{1}\left(\bar{U}\left(t-\delta_{2}\right)\right)-\kappa R_{\mu}+k_{r} C-k_{f} g(\xi, \mu) \\
& \leq U_{3}-\kappa R_{\mu}+k_{r} R_{C} \\
& \leq 0 .
\end{aligned}
$$

Finally, for the fourth coordinate let $C_{0}=0$ and $w_{0} \in Q$. Then $f_{4}^{1}\left(w_{0}\right)=k_{f} g(\xi, \mu)>0$. If $C_{0}=R_{C}$ and $w_{0} \in Q$ we have

$$
\begin{aligned}
f_{4}^{1}\left(w_{0}\right) & =k_{f} g(\xi, \mu)-k_{r} C \\
& \leq k_{f} g\left(R_{\xi}, R_{\mu}\right)-k_{r} C \\
& \leq 0
\end{aligned}
$$

By Lemma 3.6 the interval $[0, \hat{R}] \times[0, \hat{U}]$ is a positively invariant set in $\mathcal{C}^{N C R}$ under $K_{\epsilon}$. Since the above argument is true for any value of constants $R_{x}, R_{\xi}, R_{\mu}, R_{C}$ larger then those indicated, every solution starting at $w_{0} \in \mathcal{C}^{N C R}$ exists for $t \in[0, \infty]$. It also shows that all solutions enter the closed and bounded region $\left[\hat{0}, \hat{R}_{x}\right] \times\left[\hat{0}, \hat{R}_{\xi}\right] \times\left[0, R_{\mu}\right] \times\left[0, R_{C}\right] \subset \mathcal{C}^{N C R}$. This proves (a).

The proof of (b) follows directly from the decomposition (13), (15) and (16).

The proof of (c) follows from the decomposition (10) and (b).

Remark 3.8. Observe that in each case the proof of Lemma 3.7 begins with the construction of an invariant ordered interval $Q$. We will make use of these ordered intervals in some of the later proofs.

\subsection{Monotonicity}

As is indicated earlier monotonicity plays an important role in our analysis of the dynamics of nuclear gating. Thus we begin this section by recalling several essential definitions and results.

Given an order cone a function $F: C\left([-\tau, 0], \mathbf{R}^{n}\right) \rightarrow C\left([-\tau, 0], \mathbf{R}^{n}\right)$ is monotone if $\varphi \leq \psi$ implies that $F(\varphi) \leq F(\psi)$. Its differential $d F$ is strongly monotone for all $\varphi \in C\left([-\tau, 0], \mathbf{R}^{n}\right)$, if $\psi>0$ then $d F(\varphi) \psi>>0$. As is shown in [26], if $d F$ is strongly monotone, then $F$ is monotone.

Consider a system

$$
w^{\prime}=f\left(t, w_{t}\right)
$$

where $f: \mathbf{R} \times \Omega \rightarrow \mathbf{R}^{n}$, where $\Omega$ is an open subset of $C$, is continuously differentiable. The system (17) is cooperative if $\Omega$ is order convex and if $d f(w)$ satisfies

$$
d f_{i}(t, w) \varphi \geq 0 \text { whenever } \varphi \geq 0 \text { and } \varphi_{i}(0)=0
$$

for every $w \in \Omega$ and all $t$. If the system is cooperative, then by [26, Lemma 5.1.2], the derivative $d f(t, w)$ can be represented as

$$
d f_{i}(t, w) \varphi=a_{i}(t, w) \varphi_{i}(0)+\sum_{j=1}^{n} \int_{-\tau}^{0} \varphi_{j}(\theta) d_{\theta} \eta_{i j}(t, w, \theta)
$$

where $a_{i}(t, w)$ and $\eta_{i j}(t, w, \theta)$ are continuous functions of $w \in \Omega$ and $t \in \mathbf{R}$. System (17) is cooperative and irreducible if it is cooperative and the following hold:

(i) the matrix

$$
A(t, w):=\operatorname{col}\left(d f(t, w) \hat{e}_{1}, \ldots d f(t, w) \hat{e}_{n}\right)
$$

is irreducible for every $w \in \Omega$ and $t \in \mathbf{R}$. Here $\hat{e}_{j} \in C$ is the obvious embedding of the standard unit vector $e_{j} \in \mathbf{R}^{n}$.

(ii) for every $j$ such that the $j$-th variable is delayed in the $i$-th equation, we have

$$
\eta_{i j}(t, w)([-\tau,-\tau+\epsilon))>0
$$

for all small $\epsilon>0$ and all $t \in \mathbf{R}$. 
Theorem 3.9. ([8, Theorem 2.12]) Consider an r-periodic system defined on $\left.C([-\tau, 0]), \mathbf{R}^{n}\right)$

$$
w^{\prime}=f\left(t, w_{t}\right)
$$

and assume that it is cooperative and irreducible. Let $T\left(t, t_{0}, w_{0}\right)$ be a solution with the initial condition $\left.w_{0} \in C([-\tau, 0]), \mathbf{R}^{n}\right)$ at time $t$. Then the differential $d_{x} F$ of the period map

$$
F\left(w_{0}\right)=T\left(r, 0, w_{0}\right)
$$

is strongly monotone for all $\left.w_{0} \in C([-\tau, 0]), \mathbf{R}^{n}\right)$, provided $r \geq n \tau$.

Let us return to the perturbation argument for the NCR circuit. Motivated by the form of the first two equations in $(7)$, let $\bar{T}_{h}\left(t, t_{0}, w\right)$ be a solution of the system

$$
\begin{aligned}
\dot{x} & =K_{i m p}\left(\int_{-\tau}^{0} b_{\xi}(s) \xi(t+s) d s\right)-K_{\exp }(x)-\alpha x \\
\dot{\xi} & =T\left(\bar{X}\left(t-\delta_{1}\right)\right)-\gamma \xi+K_{\exp }\left(\int_{-\tau}^{0} b_{x}(s) x(t+s) d s\right)-K_{i m p}(\xi) \\
& +h(x, \xi, t)
\end{aligned}
$$

where $h$ is a fixed function uniformly $C^{1}$-close to zero, i.e.

$$
\|h(x, \xi, t)\|_{C^{1}}<\epsilon .
$$

Let

$$
F_{h}(w):=\bar{T}_{h}(r, 0, w)
$$

be the period $r$ map of (21). Observe that all functions in the system (21) are smooth. Thus, the solution maps $\bar{T}_{h}\left(t, t_{0}, w_{0}\right)$ are smooth and hence the maps $F_{h}$ are smooth.

We remark for future reference that the derivative $d f$ of the right hand side of (21) is

$$
d f(x, \xi)=\left[\begin{array}{cc}
-\frac{d K_{e x p}}{d x}-\alpha & \frac{d K_{i m p}}{d \xi} \\
\frac{d K_{e x p}}{d x}+\frac{d h}{d x} & -\gamma-\frac{d K_{i m p}}{d \xi}+\frac{d h}{d \xi}
\end{array}\right] .
$$

Proposition 3.10. System (21) is cooperative and irreducible.

Proof. We show that (21) is cooperative using the cone $\mathcal{E}^{+}$which is clearly order convex.

Consider the derivative $d f$ in (24). By assumption

$$
\frac{d K_{i m p}}{d z}>0 \text { and } \frac{d K_{\text {exp }}}{d z}>0 \text { for } z \geq 0 .
$$

For any ordered interval $[\hat{0}, \hat{R}]$ the argument of the function $K_{\exp }$ is bounded. Therefore there exists a constant $\beta$ such that $\frac{d K_{\text {exp }}}{d z} \geq \beta>0$ for all $z \in[\hat{0}, \hat{R}]$. Since the function $h(x, \xi)$ is $C^{1}$ uniformly close to zero, there exists $\epsilon_{1}$ such that for all $\epsilon<\epsilon_{1}$ we have

$$
\frac{d K_{e x p}}{d x}+\frac{d h}{d x}>0
$$

Take now $\psi=\left(\psi_{x}, \psi_{\xi}\right) \in \mathcal{E}^{+}$i.e both components are nonnegative. We want to verify (18). Take $i=1$. Then

$$
d f_{1}(w)\left(0, \psi_{\xi}\right)^{T}=\frac{d K_{i m p}}{d z} \psi_{\xi}>0
$$

since the coefficient $\frac{d K_{i m p}}{d \xi}>0$. For $i=2$ we get

$$
d f_{2}(w)\left(\psi_{x}, 0\right)^{T}=\left(\frac{d K_{e x p}}{d x}+\frac{d h}{d x}\right) \psi_{x}>0
$$


Therefore system (21) is cooperative.

Recall that a $2 \times 2$ matrix $A$ is irreducible if the off-diagonal entries are not zero. Since $D F_{\epsilon}$ satisfies this property, this verifies $(i)$ of the definition of irreducibility.

To verify $(i i)$ it is enough to observe that $\eta_{12}=\eta_{21}=\delta_{-\tau}$ a delta function at $-\tau$.

Corollary 3.11. The differential $D F_{h}$ is strongly monotone.

Proof. Apply Theorem 3.9 and Proposition 3.10.

Lemma 3.12. $D F_{0}$ is strongly positive.

Proof. This follows from [26, Lemma 3.2] which requires that three conditions (K), (I), and (R) be satisfied. In this case, the cooperativity of (21) (Proposition 3.10) is equivalent to condition (K), and the irreducibility of (21) (Proposition 3.10) implies condition (I), and since $\eta_{12}=\eta_{21}=\delta_{-\tau}$, a delta function at $-\tau$, condition $(\mathrm{R})$ is satisfied.

Lemma 3.13. The maps $F_{h}$ are monotone.

Proof. By Proposition 3.10 and [26, Lemma 5.3.3] $f$ is quasi-monotone, that is

$$
\text { if } \varphi \leq \psi \text { and } \varphi_{i}(0)=\psi_{i}(0) \text { for some } i \text {, then } f_{i}(t, \varphi) \leq f_{i}(t, \psi) \text {. }
$$

By [26, Theorem 5.1.1] quasi-monotonicity of $f$ implies that whenever $\varphi \leq \psi$ then $\bar{T}_{h}(t, 0, \varphi) \leq \bar{T}_{h}(t, 0, \psi)$ for any $t \geq 0$. Since $F_{h}(\cdot)=\bar{T}_{h}(r, 0, \cdot)$ this proves the monotonicity of $F_{h}$.

We collect these immediate results in the following Lemma.

Lemma 3.14. a. Given an ordered interval $Q_{0} \subset \mathcal{E}^{+}$from Lemma 3.7.c (see Remark 3.8) and $r \geq 2 \tau$, there is an $\epsilon_{1}$ such that the maps $F_{h}$ with $\|h\|_{C^{1}} \leq \epsilon$ and $\epsilon \in\left[0, \epsilon_{1}\right)$ are $C^{2}$, monotone maps with a differential $D F_{h}$ that is strongly monotone with respect to the cone $\mathcal{E}^{+}$. Furthermore, $D F_{0}$ is strongly positive.

b. The maps $F_{x}=F_{0}$ and $F_{u}$ are $C^{2}$, one-to-one, and monotone with differentials $D F_{x}$ and $D F_{u}$ that are each strongly monotone with respect to the cone $\mathcal{E}^{+}$, provided $r \geq 2 \tau$.

Proof. (a). follows from the previous four results.

(b). All argument of (a) apply to the case $h \equiv 0$ and thus to $F_{0}$ and $D F_{0}$. Since $F_{x}=F_{0}$ and $F_{u}$ is a time $r$ map of a completely analogous system to system (12), the results follow.

\subsection{Convergence for the open gate: $k_{f}=0$}

We begin this section with a key result concerning the global dynamics of (12) when $k_{f}=0$ and conclude with the proof of Theorem 1.3.a.

Theorem 3.15. Let $z_{0}:=\left(x_{0}, \xi_{0}\right) \succ\left(x_{1}, \xi_{1}\right)=: z_{1}$ be two initial conditions in $Q_{0}$ that are ordered by the order induced by $\mathcal{E}^{+}$. Then

$$
\lim _{n \rightarrow \infty}\left\|F_{0}^{n}\left(z_{0}\right)-F_{0}^{n}\left(z_{1}\right)\right\|=0 .
$$

Proof. By Lemma 3.7.b the map $F_{0}$ is point dissipative and completely continuous. By [11, Corollary 3.2.2] complete continuity implies that $F_{0}$ is asymptotically smooth. It follows now from [11, Theorem 2.4.6] that there is a connected global attractor $A_{0}$ under $F_{0}$ in $\mathcal{E}^{+}$.

To simplify the notation we will always take the initial time to be $t_{0}=0$. Given an initial condition $z_{0}=\left(x_{0}, \xi_{0}\right)$ at $t_{0}=0$ denote the $x$-component of $T_{0}\left(t, 0, z_{0}\right)$ by $x_{0}(t)$ and the $\xi$-component of $T_{0}\left(t, 0, z_{0}\right)$ by $\xi_{0}(t)$. Let

$$
V(x, \xi):=x+\xi
$$


The derivative of $V$ along the difference of two solutions $\left(x_{0}(t), \xi_{0}(t)\right)$ and $\left(x_{1}(t), \xi_{1}(t)\right)$ of $(12)$ is

$$
\begin{aligned}
& \dot{V}\left(x_{0}(t), \xi_{0}(t)\right)-\dot{V}\left(x_{1}(t), \xi_{1}(t)\right)=\dot{x}_{0}(t)+\dot{\xi}_{0}(t)-\dot{x}_{1}(t)-\dot{\xi}_{1}(t) \\
= & K_{i m p}\left(\int_{-\tau}^{0} b_{\xi}(s) \xi_{0}(t+s) d s\right)-K_{i m p}\left(\xi_{0}(t)\right)+K_{\exp }\left(\int_{-\tau}^{0} b_{x}(s) x_{0}(t+s) d s\right)-K_{\exp }\left(x_{0}(t)\right) \\
- & K_{i m p}\left(\int_{-\tau}^{0} b_{\xi}(s) \xi_{1}(t+s) d s\right)+K_{i m p}\left(\xi_{1}(t)\right)-K_{\exp }\left(\int_{-\tau}^{0} b_{x}(s) x_{1}(t+s) d s\right)+K_{\exp }\left(x_{1}(t)\right) \\
- & \alpha\left(x_{0}-x_{1}\right)-\gamma\left(\xi_{0}-\xi_{1}\right)
\end{aligned}
$$

Thus,

$$
\begin{aligned}
V\left(x_{0}(t), \xi_{0}(t)\right) & -V\left(x_{1}(t), \xi_{1}(t)\right)-\left(V\left(x_{0}, \xi_{0}\right)-V\left(x_{1}, \xi_{1}\right)\right) \\
& =\int_{0}^{t} \dot{V}\left(x_{0}(y), \xi_{0}(y)\right) d y-\int_{0}^{t} \dot{V}\left(x_{1}(y), \xi_{1}(y)\right) d y \\
& =B_{0}(t)-B_{1}(t)+\int_{0}^{t}-\alpha\left(x_{0}(y)-x_{1}(y)\right)-\gamma\left(\xi_{0}(y)-\xi_{1}(y)\right) d y
\end{aligned}
$$

where for $i=0,1$

$$
\begin{aligned}
B_{i}(t) & :=\int_{0}^{t} K_{i m p}\left(\int_{-\tau}^{0} b_{\xi}(s) \xi_{i}(y+s) d s\right)-K_{i m p}\left(\xi_{i}(y)\right) d y \\
& +\int_{0}^{t} K_{\exp }\left(\int_{-\tau}^{0} b_{x}(s) x_{i}(y+s) d s\right)-K_{\exp }\left(x_{i}(y)\right) d y .
\end{aligned}
$$

By assumption (H)

$$
\begin{aligned}
\int_{0}^{t} K_{i m p}\left(\int_{-\tau}^{0} b_{\xi}(s) \xi(y+s) d s\right) d y & -\int_{0}^{t} K_{i m p}(\xi(y)) d y \\
& \leq \int_{-\tau}^{t-\tau} K_{i m p}(\xi(y)) d y-\int_{0}^{t} K_{i m p}(\xi(y)) d y \\
& =\int_{-\tau}^{0} K_{i m p}(\xi(y)) d y-\int_{t-\tau}^{t} K_{i m p}(\xi(y)) d y .
\end{aligned}
$$

Choose $t=n r$ a multiple of the period of the right-hand side of (12). Observe that the $L_{1}$ norm

$$
\left|u_{t}\right|:=\int_{-\tau}^{0} u_{t}(\theta) d \theta \leq d
$$

is uniformly bounded on $Q_{0}$. Since $K_{i m p}$ is continuous, $\left|K_{i m p}\left(\xi_{t}\right)\right|$ is bounded on $Q_{0}$ as well. Therefore there exists a constant $D_{i m p}$ such that $\left|K_{i m p}\left(\xi_{t}\right)\right|-\left|K_{i m p}\left(\xi_{s}\right)\right| \leq D_{i m p}$ for all $\xi_{t}, \xi_{s} \in Q_{0}$. In particular,

$$
\begin{aligned}
\int_{0}^{n r} K_{i m p}\left(\int_{-\tau}^{0} b_{\xi}(s) \xi(y+s) d s\right) d y & -\int_{0}^{n r} K_{i m p}(\xi(y)) d y \\
& =\int_{-\tau}^{0} K_{i m p}(\xi(y)) d y-\int_{n r-\tau}^{n r} K_{i m p}(\xi(y)) d y \\
& =\left|K_{i m p}\left(\xi_{0}\right)\right|-\left|K_{i m p}\left(\xi_{t_{n r}}\right)\right| \\
& =\left|K_{i m p}\left(\xi_{0}\right)\right|-\mid K_{i m p}\left(F^{n}\left(\xi_{q}\right) \mid \leq D_{i m p}\right.
\end{aligned}
$$

where $\xi_{0} \in C\left([-\tau, 0]\right.$. A similar argument implies that there is a constant $D_{\exp }$ such that

$$
\int_{0}^{n r} K_{\exp }\left(\int_{-\tau}^{0} b_{x}(s) x_{0}(y+s) d s\right)-K_{\exp }\left(x_{0}(y)\right) d y \leq D_{\exp } .
$$


Therefore, for $i=0,1$ we have uniform bounds in $n$

$$
B_{i}(n r) \leq B
$$

where $B:=D_{\text {exp }}+D_{i m p}$. In particular,

$$
0 \leq\left|B_{0}(n r)-B_{1}(n r)\right| \leq 2 B .
$$

By Lemma 3.14.b the differential $D F_{0}$ is strongly monotone and thus $\left(x_{0}, \xi_{0}\right) \succ\left(x_{1}, \xi_{1}\right)$ implies $\left(x_{0}(t), \xi_{0}(t)\right) \succ \succ$ $\left(x_{1}(t), \xi_{1}(t)\right)$ for all $t>0$. This means that

$$
x_{0}(t)-x_{1}(t)>0 \text { and } \xi_{0}(t)-\xi_{1}(t)>0 \text { for all } t>0 .
$$

The function $V$ is continuous and so the left hand side of the equation (26) evaluated at times $n r$ with integer $n \rightarrow \infty$ is bounded uniformly on $Q_{0}$. By (28) the first two terms on the right hand side of (26) are also bounded. Therefore

$$
\beta\left(n ; z_{0}, z_{1}\right):=\int_{0}^{n r}-\alpha\left(x_{0}(y)-x_{1}(y)\right)-\gamma\left(\xi_{0}(y)-\xi_{1}(y)\right) d y
$$

is a decreasing bounded sequence. Since $z_{0}, z_{1}$ are fixed in our argument we set $\beta(n):=\beta\left(n ; z_{0}, z_{1}\right)$. It follows now that $\beta(n)$ converges and the difference

$$
\beta(n+1)-\beta(n)=-\int_{n r}^{(n+1) r} \alpha\left(x_{0}(y)-x_{1}(y)\right)+\gamma\left(\xi_{0}(y)-\xi_{1}(y)\right) d y
$$

converges to zero as $n \rightarrow \infty$. Now (29) implies

$$
x_{0}(n r)-x_{1}(n r) \rightarrow 0 \text { and } \xi_{0}(n r)-\xi_{1}(n r) \rightarrow 0
$$

as $n \rightarrow \infty$ and thus $\lim _{n \rightarrow \infty}\left\|F_{0}^{n}\left(z_{0}\right)-F_{0}^{n}\left(z_{1}\right)\right\|=0$.

The following Theorem proves the main convergence result.

Theorem 3.16. Assume the hypotheses $(\mathbf{H})$ are satisfied, $r \geq 2 \tau$ and $k_{f}=0$.

a. All solutions of (12) that start in $\mathcal{E}^{+}$, converge to a unique r-periodic solution $\Gamma\left(t, t_{0}\right)$, where $r$ is the period of the cell cycle. This solution corresponds to the fixed point of the map $F_{0}$.

b. All solutions of (9) that start in $\mathcal{B}^{+}$converge to a unique $r$-periodic solution $\Gamma\left(t, t_{0}\right)$, where $r$ is the period of the cell cycle. This solution corresponds to the fixed point of the map $H^{G}=\left(F_{x}, F_{u}\right)$.

Proof. (a). By Lemma 3.7.b the solution map $F_{0}$ is point dissipative and completely continuous. By [11, Corollary 3.2.2] complete continuity implies asymptotic smoothness. Therefore, by [11, Theorem 3.6.3] for each $\tau \in[0, r]$ there exists a global attractor $A_{0}^{\tau} \subset \mathcal{E}^{+}$and there exists an $r$-periodic solution $\Gamma\left(t, t_{0}\right)=$ $\left(x_{\Gamma}\left(t, t_{0}\right), \xi_{\Gamma}\left(t, t_{0}\right)\right)$.

The invariance of $\mathcal{E}^{+}$implies that $\Gamma\left(t, t_{0}\right) \subset \mathcal{E}^{+}$. Thus, it follows from Theorem 3.15 that solutions of (12) starting at $(x, \xi)$ with the property

$$
(x(0), \xi(0)) \succ\left(x_{\Gamma}(0), \xi_{\Gamma}(0)\right) \text { or }(x(0), \xi(0)) \prec\left(x_{\Gamma}(0), \xi_{\Gamma}(0)\right)
$$

converge to $\Gamma\left(t, t_{0}\right)$. Let $\mathcal{S}$ denote the set of such pairs of functions $(x, \xi)$. The set $\mathcal{S}$ is a strict subset of $\mathcal{E}^{+}$. However, observe that for any two functions $\left(x^{\prime}, \xi^{\prime}\right) \in \mathcal{E}^{+}$there are functions $(\bar{x}, \bar{\xi}) \in \mathcal{E}^{+}$such that $\bar{x}(\theta)>x^{\prime}(\theta), \bar{\xi}(\theta)>\xi^{\prime}(\theta)$ for all $\theta \in[-\tau, 0]$, and, at the same time, $\bar{x}(\theta)>x_{\Gamma}(\theta), \bar{\xi}(\theta)>\xi_{\Gamma}(\theta)$ for all $\theta \in[-\tau, 0]$. In other words $\left(x^{\prime}, \xi^{\prime}\right) \succ(\bar{x}, \bar{\xi})$ and $(\bar{x}, \bar{\xi})$ in $\mathcal{S}$. By Theorem 3.15 a solution starting at $\left(x^{\prime}, \xi^{\prime}\right)$ converges to the same solution as $(\bar{x}, \bar{\xi})$, that is, to $\Gamma(t, \theta)$. Since $\left(x^{\prime}, \xi^{\prime}\right) \in \mathcal{E}^{+}$was arbitrary, this finishes the proof.

(b). Applying part a. to both $F_{x}$ and $F_{u}$ separately gives convergence to $r$ periodic orbits $\Gamma_{x}\left(t, t_{0}\right)$ and $\Gamma_{u}\left(t, t_{0}\right)$, respectively. Taking $\Gamma\left(t, t_{0}\right):=\left(\Gamma_{x}\left(t, t_{0}\right), \Gamma_{u}\left(t, t_{0}\right)\right)$ finishes the proof. 
Theorem 1.3.a is now an easy corollary of Theorem 3.16.b.

Proof of Theorem 1.3.a. Recall that system (2) is asymptotic to system (5) which in turn is asymptotic to system (9). By Theorem 3.16.b the period $r$ map $H^{G}$ of system (9) satisfies the assumptions of [35, Theorem 2.4] Consequently, by [35, Theorem 2.4] all solutions of (5) converge to $\Gamma\left(t, t_{0}\right)$. By applying the same argument for systems (2) and (5) we get the final result.

\section{The open NCR gate: $0<k_{f}<<1$}

Observe that Theorem 3.16.a does not guarantee hyperbolicity of the solution $\Gamma\left(t, t_{0}\right)$. Thus, additional arguments, that are presented in this section, are necessary to obtain Theorem 1.4.a. In particular we need control not only of $K_{0}$, but also $K_{\epsilon}$.

Recall that by Lemma 3.14.b, $D F_{0}$ is a completely continuous strongly positive operator. Thus, the Krein-Rutman Theorem [26, 17] implies that the spectral radius $R:=\rho\left(D F_{0}\right)$ of $D F_{0}$ is a simple positive eigenvalue and the corresponding eigenvector $z \succ \succ 0$.

Furthermore,

$$
|\lambda|<R \text { for all } \lambda \in \sigma\left(D F_{0}\right), \text { where } \lambda \neq r .
$$

Since the operator $D F_{0}$ is completely continuous, the spectrum consists of at most a countable set of eigenvalues and the eigenvalues have no accumulation point, except possibly 0 . Therefore condition (31) implies that $R$ an isolated point of the spectrum of $D F_{0}$. Therefore by [10, Theorem 4.4] there exists a neighbourhood $N_{\delta_{0}}$ of the fixed point $Z_{0}:=\Gamma(0,0)$ and a one-dimensional $C^{1}$ center manifold $W_{0}$ of the form

$$
W_{0}=\{\zeta+q(\zeta) \mid \zeta \in \operatorname{span}\{z\} \cap N\}
$$

where $q: \operatorname{span}\{z\} \cap N_{\delta_{0}} \rightarrow \mathcal{E}^{+} \times \mu(0) \times\{0\}$ is $C^{1}$.

Furthermore, by [10, Theorem 5.1] this manifold attracts solutions in, a possibly smaller neighbourhood $N_{\delta_{1}}$, exponentially. Choose $\delta<\min \left\{\delta_{0}, \delta_{1}\right\}$ such that the neighbourhood $N_{\delta}$ also satisfies

$$
v \succ \succ Z_{0} \quad \text { or } \quad Z_{0} \succ \succ v
$$

for all $v \in W_{0} \cap N_{\delta}$. Observe that the continuity of $W_{0}$ and the fact that $q(0)=Z_{0}, q^{\prime}(0)=0$ and $z \succ \succ 0$ makes this possible.

Since $W_{0}$ is a bounded subset of a one-dimensional manifold and hence compact, by the standard persistence theory [13] there is $\epsilon_{0}>0$ such that for all $k_{f}=\epsilon<\epsilon_{0}$ there exists a $C^{1}$ manifold $W_{\epsilon}$, which is $C^{1}$-close to $W_{0}$, invariant under $K_{\epsilon}$, and attracts all solutions in $N_{\delta}$. Furthermore all points on $W_{\epsilon}$ are ordered by our choice (33).

Since $W_{\epsilon}$ is $C^{1}$-close to $W_{0}$ there are functions $f_{1}(x, \xi)$ and $f_{2}(x, \xi, t)$, with $f(x, \xi, t) r$-periodic in time $t$, satisfying

$$
(x, \xi, \mu, C) \in W_{\epsilon} \text { if and only if }\left\{\begin{array}{c}
(x, \xi, \bar{\mu}(0), 0) \in W_{0} \\
C=f_{1}(x, \xi) \\
\mu(t)-\bar{\mu}(0)=f_{2}(x, \xi, t)
\end{array}\right.
$$

and such that for all $\eta>0$ there exists an $\epsilon_{1}<\epsilon_{2}$ such that for all $\epsilon<\epsilon_{1}$

$$
\left\|f_{1}(x, \xi)\right\|_{C^{1}} \leq \eta, \quad\left\|f_{2}(x, \xi, t)\right\|_{C^{1}} \leq \eta \quad \text { for all } x, \xi \in N_{\delta}, t \in[0, r] .
$$

The dynamics on $W_{\epsilon}$ is described by

$$
\begin{aligned}
\dot{x}= & K_{i m p}\left(\int_{-\tau}^{0} b_{\xi}(s) \xi(t+s) d s\right)-K_{\exp }(x)-\alpha x \\
\dot{\xi}= & T\left(\bar{X}\left(t-\delta_{1}\right)\right)-\gamma \xi-k_{f} g\left(\xi, \mu_{0}+f_{2}(x, \xi)\right)+k_{r} f_{1}(x, \xi)+ \\
& K_{\exp }\left(\int_{-\tau}^{0} b_{x}(s) x(t+s) d s\right)-K_{i m p}(\xi) \\
\mu(t)= & \bar{\mu}(0)+f_{2}(x, \xi, t) \\
C= & f_{1}(x, \xi) .
\end{aligned}
$$


Since the dynamics is determined by the first two equations, it is described by the map $F_{\epsilon}$ given as a period map for (12), where we set

$$
h(x, \xi, t):=-k_{f} g\left(\xi, \mu_{0}+f_{2}(x, \xi, t)\right)+k_{r} f_{1}(x, \xi) .
$$

Since $\|h(x, \xi, t)\| \leq \epsilon\|g\|+\eta(\epsilon) k_{r}$, given arbitrary $\bar{\eta}>0$ there is $\epsilon_{2}<\epsilon_{1}$ such that for all $\epsilon \leq \epsilon_{2}$

$$
\|h(x, \xi, t)\| \leq \bar{\eta}
$$

Recall that (23) defines the map $F_{h}$. Setting $k_{f}=\epsilon$ in (36) uniquely defines $h$ in terms of $\epsilon$ and so in what follows we shall use the notation $F_{\epsilon}$ to denote the map $F_{h}$ with $h$ given by (36) with $k_{f}=\epsilon$.

Observe that $K_{\epsilon}$ is a perturbation of $K_{0}=\left(F_{0}, E_{0}\right)$ and $F_{\epsilon}$ is a perturbation of $F_{0}$. Furthermore, $F_{\epsilon}$ is the restriction of $K_{\epsilon}$ to the 1-dimensional manifold $W_{\epsilon}$ and $F_{0}$ is the restriction of $K_{0}$ to $W_{0}$.

Fix $\epsilon \in\left[0, \epsilon_{2}\right)$. We show that all solutions of $F_{\epsilon}$ starting in $N_{\delta}$ converge to a unique fixed point $Z_{\epsilon} \in W_{\epsilon}$ by (essentially) repeating the argument in Theorem 3.15. If $w_{0}:=\left(x_{0}, \xi_{0}, \mu_{0}, C_{0}\right)$ is an initial condition at $t_{0}=0$ we denote the $x$-component of $T_{\epsilon}\left(t, 0, w_{0}\right)$ by $x_{0}(t)$ and the $\xi$-component of $T_{\epsilon}\left(t, 0, w_{0}\right)$ by $\xi_{0}(t)$.

Theorem 4.1. There exists $\epsilon_{3}$ such that for all $\epsilon<\epsilon_{3}$ there is a fixed point $Z_{\epsilon} \in W_{\epsilon}$ of $F_{\epsilon}$, such that for any $z \in W_{\epsilon} \cap N_{\delta}$

$$
\lim _{n \rightarrow \infty} F_{\epsilon}^{n}(z)=Z_{\epsilon} .
$$

Proof. Let $z_{0}:=\left(x_{0}, \xi_{0}, \mu_{0}, C_{0}\right) \in W_{\epsilon}$ and $z_{1}:=\left(x_{1}, \xi_{1}, \mu_{1}, C_{1}\right) \in W_{\epsilon}$ be two initial conditions such that their projections $\left(x_{0}, \xi_{0}\right) \succ\left(x_{1}, \xi_{1}\right)$ are ordered by the order induced by $\mathcal{C}_{1}^{+}$. We first show that

$$
\lim _{n \rightarrow \infty}\left\|F_{\epsilon}^{n}\left(z_{0}\right)-F_{\epsilon}^{n}\left(z_{1}\right)\right\|=0 .
$$

Let $V(x, \xi):=x+\xi$. The derivative of $V$ along the difference of two solutions $z_{0}(t)=\left(x_{0}(t), \xi_{0}(t), \mu_{0}(t), C_{0}(t)\right) \in$ $W_{\epsilon}$ and $z_{1}(t)=\left(x_{1}(t), \xi_{1}(t), \mu_{1}(t), C_{1}(t)\right) \in W_{\epsilon}$ of (35) is

$$
\begin{aligned}
\dot{V}\left(z_{0}(t)\right)-\dot{V}\left(z_{1}(t)\right)= & \dot{x}_{0}(t)+\dot{\xi}_{0}(t)-\dot{x}_{1}(t)-\dot{\xi}_{1}(t) \\
= & K_{i m p}\left(\int_{-\tau}^{0} b_{\xi}(s) \xi_{0}(t+s) d s\right)-K_{i m p}\left(\xi_{0}(t)\right) \\
& +K_{\exp }\left(\int_{-\tau}^{0} b_{x}(s) x_{0}(t+s) d s\right)-K_{\exp }\left(x_{0}(t)\right) \\
& -K_{i m p}\left(\int_{-\tau}^{0} b_{\xi}(s) \xi_{1}(t+s) d s\right)+K_{i m p}\left(\xi_{1}(t)\right) \\
& -K_{\exp }\left(\int_{-\tau}^{0} b_{x}(s) x_{1}(t+s) d s\right)+K_{\text {exp }}\left(x_{1}(t)\right) \\
& -k_{f} g\left(\xi, \mu_{0}+f_{2}(x, \xi)\right)+k_{r} f_{1}(x, \xi) \\
& -\alpha\left(x_{0}-x_{1}\right)-\gamma\left(\xi_{0}-\xi_{1}\right)
\end{aligned}
$$

Thus,

$$
\begin{aligned}
V\left(z_{0}(t)\right)- & V\left(z_{1}(t)\right)-\left(V\left(z_{0}(0)\right)-V\left(z_{1}(0)\right)\right) \\
= & \int_{0}^{t} \dot{V}\left(x_{0}(z), \xi_{0}(z)\right) d z-\int_{0}^{t} \dot{V}\left(x_{1}(z), \xi_{1}(z)\right) d z \\
= & B_{0}(t)-B_{1}(t)+\int_{0}^{t}-\alpha\left(x_{0}(z)-x_{1}(z)\right)-\gamma\left(\xi_{0}(z)-\xi_{1}(z)\right) d z \\
& -k_{f} \int_{0}^{t}\left(g\left(\xi_{0}, \bar{\mu}(0)+f_{2}\left(x_{0}, \xi_{0}, t\right)\right)-g\left(\xi_{1}, \bar{\mu}(0)+f_{2}\left(x_{1}, \xi_{1}\right)\right) d z\right. \\
& +k_{r} \int_{0}^{t} f_{1}\left(x_{0}, \xi_{0}\right)-f_{1}\left(x_{1}, \xi_{1}\right) d z
\end{aligned}
$$


where $B_{0}(t)$ and $B_{1}(t)$ are defined as in Lemma 3.15 .

We estimate the last two terms in (37). First the term with $k_{f}$ is equal to

$$
\begin{aligned}
& k_{f} \int_{0}^{t} \int_{0}^{1}\left(\frac{d g}{d \xi}+\frac{d g}{d \mu} \frac{d f_{2}}{d \xi}\right)\left(s \xi_{0}+(1-s) \xi_{1}, \bar{\mu}(0)+f_{2}\left(x_{0}, s \xi_{0}+(1-s) \xi_{1}, z\right)\right) d s\left(\xi_{0}-\xi_{1}\right) d z \\
+ & k_{f} \int_{0}^{t} \int_{0}^{1} \frac{d g}{d \mu} \frac{d f_{2}}{d x}\left(\xi_{1}, \bar{\mu}(0)+f_{2}\left(s x_{0}+(1-s) x_{1}, \xi_{1}, z\right)\right) d s\left(x_{0}-x_{1}\right) d z .
\end{aligned}
$$

The last term in (37) can be written as

$$
\begin{gathered}
k_{r} \int_{0}^{t} \int_{0}^{1} \frac{d f_{1}}{d \xi}\left(s \xi_{0}+(1-s) \xi_{1}, x_{0}\right) d s\left(\xi_{0}-\xi_{1}\right) d z \\
\left.+\quad k_{r} \int_{0}^{t} \int_{0}^{1} \frac{d f_{1}}{d x}\left(\xi_{1}, s x_{0}+(1-s) x_{1}\right)\right) d s\left(x_{0}-x_{1}\right) d z .
\end{gathered}
$$

Using the notation

$$
\begin{aligned}
& B_{2}(z):=k_{f} \int_{0}^{1}\left(\frac{d g}{d \xi}+\frac{d g}{d \mu} \frac{d f_{2}}{d \xi}\right)\left(s \xi_{0}+(1-s) \xi_{1}, \bar{\mu}(0)+f_{2}\left(x_{0}, s \xi_{0}+(1-s) \xi_{1}, z\right)\right) d s \\
& B_{3}(z):=k_{f} \int_{0}^{1} \frac{d g}{d \mu} \frac{d f_{2}}{d x}\left(\xi_{1}, \bar{\mu}(0)+f_{2}\left(s x_{0}+(1-s) x_{1}, s \xi_{0}+(1-s) \xi_{1}, z\right)\right) d s \\
& B_{4}(z):=k_{r} \int_{0}^{1} \frac{d f_{1}}{d \xi}\left(s \xi_{0}+(1-s) \xi_{1}, x_{0}\right) d s \\
& \left.B_{5}(z):=k_{r} \int_{0}^{1} \frac{d f_{1}}{d x}\left(\xi_{1}, s x_{0}+(1-s) x_{1}\right)\right) d s
\end{aligned}
$$

equation (37) takes the form

$$
\begin{aligned}
& V\left(x_{0}(t), \xi_{0}(t)\right)-V\left(x_{1}(t), \xi_{1}(t)\right)-\left(V\left(x_{0}, \xi_{0}\right)-V\left(x_{1}, \xi_{1}\right)\right) \\
= & B_{0}(t)-B_{1}(t) \\
& +\int_{0}^{t}\left[B_{3}(z)-B_{5}(z)-\alpha\right]\left(x_{0}(z)-x_{1}(z)\right)+\left[B_{2}(z)-B_{4}(z)-\gamma\right]\left(\xi_{0}(z)-\xi_{1}(z)\right) d z
\end{aligned}
$$

Choose $t=n r$ a multiple of the period of the right-hand side of (35). Since $W_{\epsilon} \cap N_{\delta}$ is compact, the $L_{1}$ norm

$$
\left|v_{t}\right|:=\int_{-\tau}^{0} v_{t}(\theta) d \theta \leq d
$$

is uniformly bounded for all $v_{t} \in W_{\epsilon}$. Then the identical argument to that in Lemma 3.15 shows that there is a constant $B$ such that

$$
0 \leq\left|B_{0}(n r)-B_{1}(n r)\right| \leq 2 B .
$$

Now we estimate the terms $B_{i}(t), i=2,3,4,5$. Since $f_{1}$ and $f_{2}$ are $C^{1}$ close to zero (see (34)) and $k_{f}=\epsilon$, there is $\epsilon_{3}$ such that for $\epsilon<\epsilon_{3}$, the terms $\left|B_{3}(t)-B_{5}(t)\right|<C \epsilon$ and $\left|B_{2}(t)-B_{4}(t)\right|<C \epsilon$ are uniformly (in $t$ ) small in the set $U_{\epsilon}$. Therefore for $\epsilon \leq \epsilon_{3}$ we have that

$$
B_{3}(t)-B_{5}(t)-\alpha<0 \text { and } B_{2}(t)-B_{4}(t)-\gamma<0
$$

for all $t \geq 0$.

By Lemma 3.14.a the differential $D F_{\epsilon}$ is strongly monotone and thus $\left(x_{0}, \xi_{0}\right) \succ\left(x_{1}, \xi_{1}\right)$ implies $\left(x_{0}(t), \xi_{0}(t)\right) \succ \succ$ $\left(x_{1}(t), \xi_{1}(t)\right)$ for all $t>0$. This means that

$$
x_{0}(t)-x_{1}(t)>0 \text { and } \xi_{0}(t)-\xi_{1}(t)>0 \text { for all } t>0 .
$$


The function $V$ is continuous and so the left hand side of the equation (37) evaluated at times $n r$ with integer $n \rightarrow \infty$ is bounded on a compact set $W_{\epsilon} \cap N_{\delta}$. By (38) the first two terms on the right hand side of (37) are also bounded. Therefore

$$
\beta(n):=\int_{0}^{n r}\left[B_{3}(z)-B_{5}(z)-\alpha\right]\left(x_{0}(z)-x_{1}(z)\right)+\left[B_{2}(z)-B_{4}(z)-\gamma\right]\left(\xi_{0}(z)-\xi_{1}(z)\right) d z
$$

is a decreasing bounded sequence. Therefore $\beta(n)$ converges and the difference

$$
\beta(n+1)-\beta(n)=\int_{n r}^{(n+1) r}\left[B_{3}(z)-B_{5}(z)-\alpha\right]\left(x_{0}(z)-x_{1}(z)\right)+\left[B_{2}(z)-B_{4}(z)-\gamma\right]\left(\xi_{0}(z)-\xi_{1}(z)\right) d z
$$

converges to zero as $n \rightarrow \infty$. Now (39) implies

$$
x_{0}(n r)-x_{1}(n r) \rightarrow 0 \text { and } \xi_{0}(n r)-\xi_{1}(n r) \rightarrow 0
$$

as $n \rightarrow \infty$ and therefore $\lim _{n \rightarrow \infty}\left\|F_{\epsilon}^{n}\left(z_{0}\right)-F_{\epsilon}^{n}\left(z_{1}\right)\right\|=0$ for any pair $z_{0}>>z_{1} \in W_{\epsilon}$. Since all points in $W_{\epsilon} \cap N_{\delta}$ are ordered and $W_{\epsilon}$ is one dimensional, this implies the statement of the Theorem.

Theorem 4.2. Assume hypothesis $(\mathbf{H})$ holds and $r \geq 2 \tau$. There is an $\epsilon_{0}$ such that for all $k_{f}=\epsilon \leq \epsilon_{0}$ all solutions of (5) that start in $\mathcal{C}$, converge to a unique $r$-periodic solution $\Gamma_{\epsilon}\left(t, t_{0}\right)$, where $r$ is the period of the cell cycle.

Proof. Let $u_{\epsilon}(t)$ be the $r$-periodic solution corresponding to the fixed point $Z_{\epsilon}$ of the map $F_{\epsilon}$. We will prove the Theorem, if we show that for all $w \in \mathcal{C}$ we have

$$
\lim _{n \rightarrow \infty} K_{\epsilon}^{n}(w)=Z_{\epsilon} .
$$

Recall that the restriction of $K_{\epsilon}$ to $W_{\epsilon}$ is denoted by $F_{\epsilon}$. By [10, Theorem 5.1] the solutions $K_{\epsilon}^{n}(w)$ with $w \in N_{\delta}$ converge exponentially to a solution $y \in W_{\epsilon}$, and by Theorem $4.1 F_{\epsilon}^{n}(y) \rightarrow Z_{\epsilon}$ for all $y \in W_{\epsilon}$. By Lemma 3.7.a all solutions $K_{\epsilon}^{n}(w)$ eventually enter the set $Q=[0, \hat{R}] \times[0, \hat{U}]$. Therefore all we need to show that there is an $\epsilon_{0}$ such that for all $\epsilon<\epsilon_{0}$, solutions $K_{\epsilon}^{n}(w)$ starting at arbitrary $w \in Q$, enter the set $N_{\delta}$. To do that we show that for all $w \in Q$ there exists an $n$, independent on $w$, such that

$$
K_{0}^{n}(w) \in N_{\delta / 2} .
$$

Then, since the perturbation (22) is uniform in $w$, it will follow that given $\delta$ there is an $\epsilon_{0}$ such that for all $\epsilon<\epsilon_{0}$

$$
\left\|K_{\epsilon}^{n}(w)-K_{0}^{n}(w)\right\|<\delta / 2 \text { for all } w \in Q
$$

which implies $K_{\epsilon}^{n}(w) \in N_{\delta}$ for all $w \in Q$.

Since $K_{0}(w)$ decomposes as $K_{0}(w)=\left(F_{0}(w), E_{0}(w)\right)$ and the iterations of $E_{0}$ converge to zero exponentially and uniformly in $Q$, this is equivalent to showing that for all $w \in Q_{0}=[0, \hat{R}]$ there exists an $n$, independent on $w$, such that

$$
F_{0}^{n}(w) \in N_{\delta / 2} \cap Q_{0} .
$$

Thus to show (40) it is sufficient to prove the uniform condition (41).

From Theorem 3.15 it follows that the sequence $\beta\left(n ; z_{0}, z_{1}\right)$ defined analogously as in (30) is decreasing and bounded uniformly in $z_{0}$ and $z_{1}$. Therefore for all $\eta$ there is an $n_{0}$ such that for $n \geq n_{0}$

$$
\beta\left(n ; z_{0}, z_{1}\right)-\beta\left(n+1 ; z_{0}, z_{1}\right)=\int_{n r}^{(n+1) r} \alpha\left(x_{0}-x_{1}\right)+\gamma\left(\xi_{0}-\xi_{1}\right)<\eta
$$

and this is uniform in $z_{0}, z_{1}$.

Let $v_{0}:=\left(x_{0}, \xi_{0}\right) \in Q_{0}$ be an arbitrary point, $Z_{0}:=\left(x_{1}, \xi_{1}\right) \in Q_{0}$ be the fixed point of $F_{0}$, and let $v_{2}:=\left(x_{2}, \xi_{2}\right) \in Q_{0}$ be such that $v_{0}<<v_{2}$ and $Z_{0}<<v_{2}$. In the following estimate we denote by $\|\cdot\|$ 
the Manhattan metric in $\mathcal{C}_{1}=C^{1}\left([-\tau, 0], \mathbf{R}^{2}\right)$ i.e. ||$a-b||=\left|a_{1}-b_{1}\right|+\left|a_{1}-b_{2}\right|$ where $|\cdot|$ is norm in $C^{1}([-\tau, 0], \mathbf{R})$. We claim the following sequence of inequalities hold.

$$
\begin{aligned}
\| F_{0}^{n}\left(v_{0}\right) \leq & Z_{0}\|\leq\| F_{0}^{n}\left(v_{0}\right)-F_{0}^{n}\left(v_{2}\right)\left\|+|| F_{0}^{n}\left(v_{2}\right)-Z_{0}\right\| \\
\leq & \left|\left(x_{0}(n r)-x_{2}(n r)\right)\right|+\left|\left(\xi_{0}(n r)-\xi_{2}(n r)\right)\right|+\left|\left(x_{2}(n r)-x_{1}(n r)\right)\right|+\mid\left(\xi_{2}(n r)-\xi_{1}(n r) \mid\right. \\
\leq & \frac{1}{\min \{\alpha, \gamma\}} \alpha\left|\left(x_{0}(n r)-x_{2}(n r)\right)\right|+\gamma\left|\left(\xi_{0}(n r)-\xi_{2}(n r)\right)\right| \\
& +\frac{1}{\min \{\alpha, \gamma\}} \alpha\left|\left(x_{2}(n r)-x_{1}(n r)\right)\right|+\gamma \mid\left(\xi_{2}(n r)-\xi_{1}(n r) \mid\right. \\
\leq & \frac{D}{\min \{\alpha, \gamma\}} \int_{n r}^{(n+1) r} \alpha\left(x_{0}(t)-x_{2}(t)\right)+\gamma\left(\xi_{0}(t)-\xi_{2}(t)\right) d t \\
& +\frac{D}{\min \{\alpha, \gamma\}} \int_{n r}^{(n+1) r} \alpha\left(x_{2}(n r)-x_{1}(n r)\right)+\gamma\left(\xi_{2}(n r)-\xi_{1}(n r)\right. \\
\leq & \frac{2 \eta D}{\min \{\alpha, \gamma\}},
\end{aligned}
$$

for $n \geq n_{0}$ by (42). The constant $D$ is computed by the following argument. Set $g(t):=\alpha\left(x_{0}(t)-x_{2}(t)\right)+$ $\gamma\left(\xi_{0}(t)-\xi_{2}(t)\right)$. Then

$$
\begin{aligned}
g(n r) & =\left(\frac{g(n r)}{r \min _{s \in[n r,(n+1) r]} g(s)}\right) \int_{n r}^{(n+1) r} \min g(t) d t \\
& \leq\left(\frac{g(n r)}{r \min _{s \in[n r,(n+1) r]} g(s)}\right) \int_{n r}^{(n+1) r} g(t) d t .
\end{aligned}
$$

The differences $z_{1}:=x_{0}(t)-x_{2}(t)$ and $z_{2}:=\xi_{0}(t)-\xi_{2}(t)$ solve linear non-autonomous system of differential equations

$$
\begin{aligned}
& \dot{z}_{1}=\int_{0}^{1} \frac{d K_{i m p}}{d \xi}\left(s \xi_{0}+(1-s) \xi_{2}\right) d s z_{2}-\int_{0}^{1} \frac{d K_{\text {exp }}}{d x}\left(s x_{0}+(1-s) x_{2}\right) d s z_{1}-\alpha z_{1} \\
& \dot{z}_{2}=-\gamma z_{2}+\frac{d K_{\text {exp }}}{d x}\left(s x_{0}+(1-s) x_{2}\right) d s z_{1}-\frac{d K_{i m p}}{d \xi}\left(s \xi_{0}+(1-s) \xi_{2}\right) d s z_{2}
\end{aligned}
$$

The matrix on the right hand side is evaluated at a point in $Q_{0}$. Since $Q_{0}$ is bounded, this matrix is uniformly bounded on $Q_{0}$ and so by the Gronwall inequality there is a constant $E$ such that

$$
u_{1}(t) \leq E u_{1}(s) \quad \text { and } \quad u_{2}(t) \leq E u_{2}(s)
$$

for all $s \in[n r,(n+1) r]$. This implies

$$
g(n r) \leq E \min _{s \in[n r,(n+1) r]} g(s),
$$

which implies

$$
\left(\frac{g(n r)}{r \min _{s \in[n r,(n+1) r]} g(s)}\right) \leq E / r=: D .
$$

This finishes the proof of the estimate (43). Since $\eta$ is arbitrary, the estimate (43) proves (41) and thus the Theorem.

Proof of Theorem 1.4.a Fix $\epsilon \in\left[0, \epsilon_{0}\right)$, where $\epsilon_{0}$ is determined by the Theorem 4.2. Recall that system (3) is asymptotic to system (7). By Theorem 4.2 the map $K_{\epsilon}$ satisfies the assumptions of [35, Theorem 2.4] and since $K_{\epsilon}$ has the unique fixed point $Z_{\epsilon}$, all solutions of (3) converge to the corresponding periodic orbit $\Gamma_{\epsilon}\left(t, t_{0}\right)$. 


\section{The closed gate: $k_{r}=0$}

As the slight asymmetry in Theorems 1.3 and 1.4 suggest, the proof for the closed gate is similar in spirit, though not identical, that of the open gate. Thus in our analysis of the case $k_{r}=0$ we concentrate on its differences from the setting of $k_{f}=0$.

For $k_{r}=0$ the system (5) reduces to

$$
\begin{aligned}
\dot{x}= & K_{\text {imp }}^{0}\left(\int_{-\tau}^{0} b_{\xi}(s) \xi(t+s) d s\right)-K_{\text {exp }}^{0}(x)-\alpha x \\
\dot{\xi}= & T^{0}\left(\bar{X}\left(t-\delta_{1}\right)\right)-\gamma \xi-k_{f} g(\xi, \mu)+ \\
& K_{\text {exp }}^{0}\left(\int_{-\tau}^{0} b_{x}(s) x(t+s) d s\right)-K_{\text {imp }}^{0}(\xi) \\
\dot{u}= & K_{\text {imp }}^{1}\left(\int_{-\tau}^{0} b_{\mu}(s) \mu(t+s) d s\right)-K_{\text {exp }}^{1}(u)-\alpha u \\
\dot{\mu}= & T^{1}\left(\bar{U}\left(t-\delta_{2}\right)\right)-\kappa \mu-k_{f} g(\xi, \mu)+ \\
& K_{\text {exp }}^{1}\left(\int_{-\tau}^{0} b_{u}(s) u(t+s) d s\right)-K_{i m p}^{1}(\mu) \\
\dot{C}= & k_{f} g(\xi, \mu) .
\end{aligned}
$$

Observe that (44) decomposes into the first four equations

$$
\begin{aligned}
\dot{x}= & K_{i m p}^{0}\left(\int_{-\tau}^{0} b_{\xi}(s) \xi(t+s) d s\right)-K_{\text {exp }}^{0}(x)-\alpha x \\
\dot{\xi}= & T^{0}\left(\bar{X}\left(t-\delta_{1}\right)\right)-\gamma \xi-k_{f} g(\xi, \mu)+ \\
& K_{\text {exp }}^{0}\left(\int_{-\tau}^{0} b_{x}(s) x(t+s) d s\right)-K_{i m p}^{0}(\xi) \\
\dot{u}= & K_{i m p}^{1}\left(\int_{-\tau}^{0} b_{\mu}(s) \mu(t+s) d s\right)-K_{\text {exp }}^{1}(u)-\alpha u \\
\dot{\mu}= & T^{1}\left(\bar{U}\left(t-\delta_{2}\right)\right)-\kappa \mu-k_{f} g(\xi, \mu)+ \\
& K_{\text {exp }}^{1}\left(\int_{-\tau}^{0} b_{u}(s) u(t+s) d s\right)-K_{i m p}^{1}(\mu)
\end{aligned}
$$

and the last one, whose solution is completely determined by the solution of (45). We denote the phase space of (45) by

$$
(x, \xi, u, \mu) \in \mathcal{B}=C^{1}\left([-\tau, 0], \mathbf{R}^{4}\right),
$$

and hence the phase space for $(44)$ is $\mathcal{B} \times \mathbf{R}$.

In addition to the positive cone $\mathcal{B}^{+}$, we make use of the cone $D_{0} \subset \mathcal{B}$ defined by

$$
D_{0}:=\left\{(x, \xi, u, \mu) \in \mathcal{B}: x_{t} \geq 0, \xi_{t} \geq 0, u_{t} \leq 0, \mu_{t} \leq 0\right\} .
$$

We denote by $G_{s}$ the time $r$ map of the reduced system (45), that is

$$
G_{s}\left(w_{0}\right):=U_{s}\left(r, 0, w_{0}\right),
$$

where $U_{s}\left(t, t_{0}, w_{0}\right)$ is the set of time $t$ mappings generated by (45). Similarly, $G_{f}$ denotes the time $r$ map of the full system (44), that is

$$
G_{f}\left(w_{0}\right):=U_{f}\left(r, 0, w_{0}\right),
$$

where $U_{f}\left(t, t_{0}, w_{0}\right)$ is the set of time $t$ mappings generated by (44).

Lemma 5.1. 1. The map $G_{s}$ is $C^{2}$, compact, monotone (with respect to $D_{0}$ ) and the differential $d G_{s}$ is strongly monotone (with respect to $D_{0}$ ). 
2. There exists a bounded set $P \subset \mathcal{B}^{+}$which is positively invariant under $G_{s}$ and all solutions starting in $\mathcal{B}^{+}$will eventually enter $P$. Therefore all solution maps $U_{s}\left(t, t_{0}, w_{0}\right)$ (in particular the map $G_{s}$ ) are point dissipative and completely continuous.

Proof. With the exception of the fact that the right hand side of (45) is

$$
d f(x, \xi, u, \mu)=\left[\begin{array}{cccc}
-\frac{d K_{\text {exp }}}{d x}-\alpha & \frac{d K_{i m p}}{d \xi} & 0 & 0 \\
\frac{d K_{\text {exp }}}{d x} & -\gamma-\frac{d K_{i m p}}{d \xi}-\frac{d g}{d \xi} & 0 & -\frac{d g}{d \mu} \\
0 & 0 & -\frac{d K_{\text {exp }}}{d u}-\eta & \frac{d K_{i m p}}{d \mu} \\
0 & -\frac{d g}{d \xi} & \frac{d K_{\text {exp }}}{d u} & -\kappa-\frac{d K_{i m p}}{d \mu}
\end{array}\right]
$$

and hence the appropriate order cone is $D_{0}$, the proof of this lemma follows from the same arguments employed in the proof of Lemma 3.7 in the case that $k_{f}=0$.

As in the case of $k_{f}=0$, to simplify the notation we present the arguments with the initial time taken to be $t_{0}=0$. If $w_{0}:=\left(x_{0}, \xi_{0}, u_{0}, \mu_{0}\right)$ is an initial condition at $t_{0}=0$ we denote the $x$-component of $U_{s}\left(t, 0, w_{0}\right)$ by $x_{0}(t)$, the $\xi$-component by $\xi_{0}(t), u$-component by $u_{0}(t)$ and the $\mu$-component by $\mu_{0}(t)$.

Theorem 5.2. Let $w_{0}:=\left(x_{0}, \xi_{0}, u_{0}, \mu_{0}\right) \succ w_{1}:=\left(x_{1}, \xi_{1}, u_{1}, \mu_{1}\right)$ be two initial conditions that are ordered by the order induced by $D_{0}$. Then

$$
\lim _{n \rightarrow \infty}\left\|G_{s}^{n}\left(z_{0}\right)-G_{s}^{n}\left(z_{1}\right)\right\|=0 .
$$

Proof. By Lemma 5.1.2 the map $G_{s}$ is point dissipative and completely continuous. By [11, Corollary 3.2.2] complete continuity implies that $G_{s}$ is asymptotically smooth. It follows now from [11, Theorem 3.6.3] that there is a connected global attractor $A$ under $G_{s}$ in $\mathcal{B}^{+}$. Since $g(\xi, \mu)$ is continuous it follows immediately that the map $G_{f}$ also admits a global attractor $A^{\prime}$ in $\mathcal{B}^{+} \times \mathbf{R}$.

We will consider two Lyapunov functions. The first is

$$
V(x, \xi, u, \mu, C)=x+\xi+C
$$

defined on the space $\mathcal{B}^{+} \times \mathbf{R}$. As in the proof of Theorem 3.15 we will evaluate this function along the difference of two solutions whose initial conditions are ordered with respect to the cone $D_{0} \times \mathbf{R}^{+}$.

The derivative of $V$ along the difference of two solutions

$$
w_{i}(t):=\left(x_{i}(t), \xi_{i}(t), u_{i}(t), \mu_{i}(t), C_{i}(t)\right), \quad i=0,1
$$

of $(44)$ is

$$
\begin{aligned}
\dot{V}\left(w_{0}(t)\right)-\dot{V}\left(w_{0}(t)\right)= & \dot{x}_{0}(t)+\dot{\xi}_{0}(t)+\dot{C}_{0}(t)-\dot{x}_{1}(t)-\dot{\xi}_{1}(t)-\dot{C}_{1}(t) \\
= & K_{i m p}\left(\int_{-\tau}^{0} b_{\xi}(s) \xi_{0}(t+s) d s\right)-K_{i m p}\left(\xi_{0}(t)\right) \\
& +K_{\exp }\left(\int_{-\tau}^{0} b_{x}(s) x_{0}(t+s) d s\right)-K_{\exp }\left(x_{0}(t)\right) \\
& -K_{i m p}\left(\int_{-\tau}^{0} b_{\xi}(s) \xi_{1}(t+s) d s\right)+K_{i m p}\left(\xi_{1}(t)\right) \\
& -K_{\exp }\left(\int_{-\tau}^{0} b_{x}(s) x_{1}(t+s) d s\right)+K_{\exp }\left(x_{1}(t)\right) \\
& -\alpha\left(x_{0}-x_{1}\right)-\gamma\left(\xi_{0}-\xi_{1}\right) .
\end{aligned}
$$

Notice that this is the same expression as we had in (25). Therefore

$$
\begin{aligned}
& V\left(w_{0}(t)\right)-V\left(w_{1}(t)\right)-\left(V\left(w_{0}\right)-V\left(w_{1}\right)\right) \\
= & \int_{0}^{t} \dot{V}\left(x_{0}(z), \xi_{0}(z), \mu_{0}(z), C_{0}(z)\right) d z-\int_{0}^{t} \dot{V}\left(x_{1}(z), \xi_{1}(z), \mu_{1}(z), C_{1}(z)\right) d z \\
= & B_{0}(t)-B_{1}(t)+\int_{0}^{t}-\alpha\left(x_{0}(z)-x_{1}(z)\right)-\gamma\left(\xi_{0}(z)-\xi_{1}(z)\right) d z
\end{aligned}
$$


where $B_{i}(t)$ is defined in $(27)$.

We evaluate this expression at $t=n r$, a multiple of the period, and use an argument identical to that in Theorem 3.15 to show that there is a constant $Z$ such that

$$
0 \leq\left|B_{0}(n r)-B_{1}(n r)\right| \leq Z \text { for all integers } n>0 .
$$

By Lemma 5.1 the map $G_{s}$ is strongly monotone with respect to $D_{0}$ and thus $\left(x_{0}, \xi_{0}, u_{0}, \mu_{0}\right) \succ\left(x_{1}, \xi_{1}, u_{1}, \mu_{1}\right)$ implies

$$
\left(x_{0}(t), \xi_{0}(t), u_{0}(t), \mu_{0}(t)\right)>>\left(x_{1}(t), \xi_{1}(t), u_{1}(t), \mu_{1}(t)\right)
$$

for all $t>0$. This means that, in particular, for all $t>0$

$$
x_{0}(t)-x_{1}(t)>0 \text { and } \xi_{0}(t)-\xi_{1}(t)>0 .
$$

Since the left hand side of the equation (46) is bounded on a compact attractor $A^{\prime} \in \mathcal{B}^{+} \times \mathbf{R}, \mid B_{0}(n r)$ $B_{1}(n r) \mid \leq Z$ is bounded as well, and the sequence

$$
\int_{0}^{n r}-\alpha\left(x_{0}(z)-x_{1}(z)\right)-\gamma\left(\xi_{0}(z)-\xi_{1}(z)\right) d z
$$

is a bounded monotone sequence. This implies that

$$
x_{0}(n r)-x_{1}(n r) \rightarrow 0 \text { and } \xi_{0}(n r)-\xi_{1}(n r) \rightarrow 0
$$

as $n \rightarrow \infty$.

To finish the proof of the theorem we need to show that $u_{0}(n r)-u_{1}(n r) \rightarrow 0$ and $\mu_{0}(n r)-\mu_{1}(n r) \rightarrow 0$ as $n \rightarrow \infty$. In order to do that we use an analogous Lyapunov function

$$
\bar{V}(x, \xi, \mu, u, C):=u+\mu+C .
$$

Then the same argument as above shows that the derivative of $\bar{V}$ along the difference of two solutions $w_{0}$ and $w_{1}$ of $(44)$ is

$$
\begin{aligned}
& \dot{\bar{V}}\left(w_{0}(t)\right)-\dot{\bar{V}}\left(w_{1}(t)\right) \\
= & \bar{B}_{0}(t)-\bar{B}_{1}(t)-\eta\left(u_{0}-u_{1}\right)-\kappa\left(\mu_{0}-\mu_{1}\right),
\end{aligned}
$$

where $\bar{B}_{i}(t)$ are expressions analogous to the $B_{i}(t), i=0,1$ defined above where we exchange $x$ for $u$ and $\xi$ for $\mu$. We compute as before

$$
\begin{aligned}
& \bar{V}\left(w_{0}(t)\right)-\bar{V}\left(w_{1}(t)\right)-\left(\bar{V}\left(w_{0}\right)-\bar{V}\left(w_{1}\right)\right. \\
= & \bar{B}_{0}(t)-\bar{B}_{1}(t)+\int_{0}^{t}-\eta\left(u_{0}(z)-u_{1}(z)\right)-\kappa\left(\mu_{0}(z)-\mu_{1}(z) d z .\right.
\end{aligned}
$$

Again, we evaluate this expression at multiples of the period, $t=n r$. By the previous argument $\mid \bar{B}_{0}(n r)-$ $\bar{B}_{1}(n r) \mid$ is uniformly bounded (though perhaps by a different constant) and the left hand side of (48) is uniformly bounded. By strong monotonicity of $G_{s}$ with respect to $D_{0}$ we get that $u_{1}(t)>u_{0}(t)$ and $\mu_{1}(t)>\mu_{0}(t)$ and for all $t>0$. Hence

$$
\delta(n):=\int_{0}^{n r}-\eta\left(u_{0}(s)-u_{1}(s)\right)-\kappa\left(\mu_{0}(s)-\mu_{1}(s)\right) d s
$$

is a bounded sequence of positive numbers, which implies $\delta(n+1)-\delta(n) \rightarrow 0$ as $n \rightarrow \infty$, and thus

$$
\lim _{n \rightarrow \infty}\left(u_{0}(n r)-u_{1}(n r)\right)=0 \text { and } \lim _{n \rightarrow \infty}\left(\mu_{0}(n r)-\mu_{1}(n r)\right)=0 .
$$

Now (47) and (49) imply $\lim _{n \rightarrow \infty}\left\|G_{s}^{n}\left(z_{0}\right)-G_{s}^{n}\left(z_{1}\right)\right\|=0$. 
Theorem 5.3. Assume the hypotheses $(\mathbf{H})$ are satisfied, $r \geq 3 \tau$, and $k_{r}=0$. Then all solutions of (44) that start in $\mathcal{B}^{+}$, converge to a unique r-periodic solution $\Gamma(t)$, where $r$ is the period of the cell cycle.

Proof. By Lemma 5.1.2 the solution maps $U_{s}$ generated by (44) are point dissipative and completely continuous. Since $g(\xi, \mu)$ is continuous, the maps $U_{f}$ are also point dissipative and completely continuous. By [11, Corollary 3.2.2] complete continuity implies asymptotic smoothness. Therefore we can conclude by [11, Theorem 3.6.3] that the global attractor $A_{\sigma}$ exists for each $\sigma \in[0, r]$. Furthermore, there exists an $r$-periodic solution $\Gamma\left(t, t_{0}\right)=\left(x_{\Gamma}, \xi_{\Gamma}, u_{\Gamma}, \mu_{\Gamma}, C_{\Gamma}\right)$. By the invariance of $\mathcal{B}^{+} \times \mathbf{R}$ we have $\Gamma\left(t, t_{0}\right) \subset \mathcal{B}^{+} \times \mathbf{R}$. It follows now from Theorem 5.2 that solutions of (44) starting at $(x, \xi, u, \mu, C)$ with the property

$$
\begin{array}{rll}
(x(0), \xi(0), u(0), \mu(0), C(0)) & \succ \quad\left(x_{\Gamma}(0), \xi_{\Gamma}(0), u_{\Gamma}(0), \mu_{\Gamma}(0), C_{\Gamma}(0)\right) \\
& \text { or } & \\
(x(0), \xi(0), u(0), \mu(0), C(0)) & \prec & \left(x_{\Gamma}(0), \xi_{\Gamma}(0), u_{\Gamma}(0), \mu_{\Gamma}(0), C_{\Gamma}(0)\right)
\end{array}
$$

converge to $\Gamma\left(t, t_{0}\right)$. We denote the set of such functions $(x, \xi, u, \mu, C)$ by $\mathcal{F}$. As in the proof of Theorem 3.16 we note that any point $\left(x^{\prime}, \xi^{\prime}, u^{\prime}, \mu^{\prime}, C^{\prime}\right) \in \mathcal{B}^{+} \times \mathbf{R}$ is in an ordered relationship with at least one point in $\mathcal{F}$. By Lemma 5.2 all these solutions converge to the same solution, namely $\Gamma\left(t, t_{0}\right)$.

Proof of Theorem 1.3b. By Theorem 5.3 the map $G_{f}$ satisfies assumption of the [35, Theorem 2.4]. Since solution maps of the system (3) are asymptotic to the solution maps of the system (44), [35, Theorem 2.4] implies that every solution of system (3) converges to the unique periodic orbit $\Gamma\left(t, t_{0}\right)$ of the system (44).

\subsection{The closed NCR gate: $0<k_{r}<<1$}

To prove the Theorem 1.4.b we proceed along an analogous route as in the case of Theorem 1.4.a We will only show the argument for $k_{r}=0$ since there are substantial differences with the case $k_{f}=0$ and in the interest of space we will leave the perturbation argument to the reader.

In the NCR example the system (44) becomes

$$
\begin{aligned}
\dot{x}= & K_{i m p}\left(\int_{-\tau}^{0} b_{\xi}(s) \xi(t+s) d s\right)-K_{\exp }(x)-\alpha x \\
\dot{\xi}= & T^{0}\left(\bar{X}\left(t-\delta_{1}\right)\right)-\gamma \xi-k_{f} g(\xi, \mu)+ \\
& K_{\exp }\left(\int_{-\tau}^{0} b_{x}(s) x(t+s) d s\right)-K_{i m p}(\xi) \\
\dot{\mu}= & T^{1}\left(\bar{U}\left(t-\delta_{2}\right)\right)-\kappa \mu-k_{f} g(\xi, \mu) \\
\dot{C}= & k_{f} g(\xi, \mu)
\end{aligned}
$$

the system (45) becomes

$$
\begin{aligned}
\dot{x}= & K_{i m p}\left(\int_{-\tau}^{0} b_{\xi}(s) \xi(t+s) d s\right)-K_{\exp }(x)-\alpha x \\
\dot{\xi}= & T\left(\bar{X}\left(t-\delta_{1}\right)\right)-\gamma \xi-k_{f} g(\xi, \mu)+ \\
& K_{\exp }\left(\int_{-\tau}^{0} b_{x}(s) x(t+s) d s\right)-K_{i m p}(\xi) \\
\dot{\mu}= & T\left(\bar{U}\left(t-\delta_{2}\right)\right)-\kappa \mu-k_{f} g(\xi, \mu),
\end{aligned}
$$

where we dropped the unnecessary superscripts.

We identify the phase space of (51) as

$$
(x, \xi, \mu) \in \mathcal{E} \times \mathbf{R}:=C^{1}\left([-\tau, 0], \mathbf{R}^{2}\right) \times \mathbf{R},
$$


and then the phase space for all four equations including the equation for $C$, is $\mathcal{E} \times \mathbf{R}^{2}$. Recall, that the positive cone of $\mathcal{E}$ is $\mathcal{E}^{+}$. Further, we denote a cone $D_{0}^{N C R} \subset \mathcal{E} \times \mathbf{R}$

$$
D_{0}^{N C R}:=\mathcal{E}^{+} \times \mathbf{R}^{-}=\left\{(x, \xi, \mu) \in \mathcal{E} \times \mathbf{R}: x_{t} \geq 0, \xi_{t} \geq 0, \mu \leq 0\right\} .
$$

We denote by $G_{s}^{N C R}$ the time $r$ map of the system (51), that is

$$
G_{s}^{N C R}\left(w_{0}\right):=U_{s}^{N C R}\left(r, 0, w_{0}\right)
$$

where $U_{s}^{N C R}\left(t, t_{0}, w_{0}\right)$ is the set of time $t$ mappings generated by (51). Similarly, let $G_{f}^{N C R}$ be the time $r$ map of the system (50).

The next Lemma is completely analogous to Lemma 5.1.

Lemma 5.4. 1. The map $G_{s}^{N C R}$ is $C^{2}$, compact, monotone (with respect to $D_{0}^{N C R}$ ) and the differential $d G_{s}^{N C R}$ is strongly monotone (with respect to $D_{0}^{N C R}$ ).

2. There exists a bounded set $P \subset \mathcal{E}^{+} \times \mathbf{R}^{+}$which is positively invariant under $G_{s}^{N C R}$ and all solutions starting in $\mathcal{E}^{+} \times \mathbf{R}^{+}$will eventually enter $P$. Therefore all solution maps $U_{s}^{N C R}\left(t, t_{0}, w_{0}\right)$ (in particular the map $G_{s}^{N C R}$ ) are point dissipative and completely continuous.

Next Theorem is analogous to Theorem 5.2 but the choice of a Lyapunov function is slightly different. We will point out the difference in the proof.

Theorem 5.5. Let $z_{0}:=\left(x_{0}, \xi_{0}, \mu_{0}\right) \succ z_{1}:=\left(x_{1}, \xi_{1}, \mu_{1}\right)$ be two initial conditions on $P$ that are ordered by the order induced by $D_{0}^{N C R}$. Then

$$
\lim _{n \rightarrow \infty}\left\|\left(G_{s}^{N C R}\right)^{n}\left(z_{0}\right)-\left(G_{s}^{N C R}\right)^{n}\left(z_{1}\right)\right\|=0 .
$$

Proof. By Lemma 5.4.2 the map $G_{s}^{N C R}$ is point dissipative and completely continuous. By [11, Corollary 3.2.2] complete continuity implies asymptotic smoothness. Therefore we can conclude by [11, Theorem 3.6.3] Corollary 3.2.2 of [11] that there is a connected global attractor $A$ under $G_{s}^{N C R}$ in $\mathcal{E}^{+} \times \mathbf{R}^{+}$. Since $g(\xi, \mu)$ is continuous it follows immediately that the map $G_{f}^{N C R}$ also admits a global attractor $A^{\prime}$ in $\mathcal{E}^{+} \times \mathbf{R}^{2,+}$.

We will consider two Lyapunov functions. First we consider $V(x, \xi, \mu, C)=x+\xi+C$ on the space $\mathcal{E}^{+} \times$ $\mathbf{R}^{2,+}$. This is the same function as in Theorem 5.2 and we get the identical result to (46). The derivative of $V$ along the difference of two solutions $w_{0}(t):=\left(x_{0}(t), \xi_{0}(t), \mu_{0}(t), C_{0}(t)\right)$ and $w_{1}(t):=\left(x_{1}(t), \xi_{1}(t), \mu_{1}(t), C_{1}(t)\right)$ is

$$
\begin{aligned}
& V\left(w_{0}(t)\right)-V\left(w_{1}(t)\right)-\left(V\left(w_{0}(0)\right)-V\left(w_{1}(0)\right)\right. \\
= & \int_{0}^{t} \dot{V}\left(x_{0}(z), \xi_{0}(z), \mu_{0}(z), C_{0}(z)\right) d z-\int_{0}^{t} \dot{V}\left(x_{1}(z), \xi_{1}(z), \mu_{1}(z), C_{1}(z)\right) d z \\
= & B_{0}(t)-B_{1}(t)+\int_{0}^{t}-\alpha\left(x_{0}(z)-x_{1}(z)\right)-\gamma\left(\xi_{0}(z)-\xi_{1}(z)\right) d z
\end{aligned}
$$

where $B_{0}(t)$ is defined in (27). In the same way as in Theorem 5.2 this implies

$$
x_{0}(n r)-x_{1}(n r) \rightarrow 0 \text { and } \xi_{0}(n r)-\xi_{1}(n r) \rightarrow 0
$$

as $n \rightarrow \infty$.

To finish the proof of the Theorem we still need to show that $\mu_{0}(n r)-\mu_{1}(n r) \rightarrow 0$ as $n \rightarrow \infty$. Here we have to choose a different function then in Theorem 5.2. We define $\bar{U}(x, \xi, \mu, C)=x+\xi+\mu+2 C$. Then the derivative of $\bar{U}$ along the difference of two solutions $\left(x_{0}(t), \xi_{0}(t), \mu_{0}(t), C_{0}(t)\right)$ and $\left(x_{1}(t), \xi_{1}(t), \mu_{1}(t), C_{1}(t)\right)$ of (51) with $C$ equation is

$$
\begin{aligned}
& \dot{\bar{U}}\left(\left(x_{0}(t), \xi_{0}(t), \mu_{0}(t), C_{0}(t)\right)-\dot{\bar{U}}\left(\left(x_{1}(t), \xi_{1}(t), \mu_{1}(t), C_{1}(t)\right)\right.\right. \\
= & \dot{x}_{0}(t)+\dot{\xi}_{0}(t)+\dot{\mu}_{0}(t)+2 \dot{C}_{0}(t)-\dot{x}_{1}(t)-\dot{\xi}_{1}(t)-\dot{\mu}_{1}(t)-\dot{C}_{1}(t) \\
= & B_{0}(t)-B_{1}(t)-\alpha\left(x_{0}-x_{1}\right)-\gamma\left(\xi_{0}-\xi_{1}\right)-\kappa\left(\mu_{0}-\mu_{1}\right)
\end{aligned}
$$


We compute

$$
\begin{aligned}
& \bar{U}\left(x_{0}(t), \xi_{0}(t), \mu_{0}(t), C_{0}(t)\right)-\bar{U}\left(x_{1}(t), \xi_{1}(t), \mu_{1}(t), C_{1}(t)\right) \\
- & \left(\bar{U}\left(x_{0}, \xi_{0}, \mu_{0}, C_{0}\right)-\bar{U}\left(x_{1}, \xi_{1}, \mu_{1}, C_{1}\right)\right. \\
= & \int_{0}^{t} \dot{\bar{U}}\left(x_{0}(z), \xi_{0}(z), \mu_{0}(z), C_{0}(z)\right) d z-\int_{0}^{t} \dot{\bar{U}}\left(x_{1}(z), \xi_{1}(z), \mu_{1}(z), C_{1}(z)\right) d s \\
= & B_{0}(t)-B_{1}(t)+\int_{0}^{t}-\alpha\left(x_{0}(z)-x_{1}(z)\right)-\gamma\left(\xi_{0}(z)-\xi_{1}(z)\right)-\kappa\left(\mu_{0}(z)-\mu_{1}(z) d z .\right.
\end{aligned}
$$

We evaluate $t:=n r$. By the previous argument $\left|B_{0}(n r)-B_{1}(n r)\right| \leq Z$ is uniformly bounded, the left hand side of (54) is uniformly bounded and, finally,

$$
\int_{0}^{n r}-\alpha\left(x_{0}(z)-x_{1}(z)\right)-\gamma\left(\xi_{0}(z)-\xi_{1}(z)\right) d z
$$

is bounded and converges to zero as $n \rightarrow \infty$ by (53). By the strong monotonicity of $G_{s}^{N C R}$ with respect to $D_{0}^{N C R}$ we get that $\mu_{1}(t)>\mu_{0}(t)$ for all $t>0$. Therefore $-\kappa\left(\mu_{0}(s)-\mu_{1}(s)\right) d s>0$ is positive for all $s$. Hence

$$
\delta(n):=\int_{0}^{n r}-\kappa\left(\mu_{0}(s)-\mu_{1}(s)\right) d s
$$

is a bounded sequence of positive numbers, which implies $\delta(n+1)-\delta(n) \rightarrow 0$ as $n \rightarrow \infty$, and thus

$$
\lim _{n \rightarrow \infty} \mu_{0}(n r)-\mu_{1}(n r)=0 .
$$

This with (53) implies that $\lim _{n \rightarrow \infty}\left\|\left(G_{s}^{N C R}\right)^{n}\left(z_{0}\right)-\left(G_{s}^{N C R}\right)^{n}\left(z_{1}\right)\right\|=0$.

The proof of the following Theorem is analogous to proof of Theorem 5.3.

Theorem 5.6. Assume all assumptions of Theorem 1.4. Then all solutions of (50) that start in $\mathcal{B}^{N C R,+}$, converge to a unique $r$-periodic solution $\Gamma\left(t, t_{0}\right)$, where $r$ is the period of the cell cycle.

Proof of Theorem 1.4b.

By Theorem 5.6 the map $G_{f}^{N C R}$ satisfies assumption of the [35, Theorem 2.4]. Since solution maps of the system (3) are asymptotic to the solution maps of the system (50), [35, Theorem 2.4] implies that every solution of system (3) converges to the unique periodic orbit $\Gamma\left(t, t_{0}\right)$ of the system $(50)$.

\section{A concluding query}

As is demonstrated in Section 3.5, given a fixed initial time and initial condition, the asymptotic dynamics under the assumption that $k_{f}=0$ appears to be qualitatively the same for the general gate and the NCR gate; in both cases the solution tends toward a periodic orbit. The results in Sections 4 and 5.1 guarantee that the periodic orbit for the NCR gate does not bifurcate under perturbations in the reaction rates for the formation and disassociation of the complex $C$. For the general gate, however, the situation is different.

While the perturbation to the NCR gate equations and to the general gate equations involves the same variables $x$ and $\xi$, the latter couples two different systems, described by the maps $F_{x}$ and $F_{u}$, together. As a consequence the analogy of Lemma 3.14.a does not hold. The fundamental reason is that the derivative of the right hand side of (9) is block diagonal, while the corresponding derivative of the perturbed system is not. In contrast in NCR gate equations the derivative of the original and perturbed system have the same structure, see (24). In other words, the perturbation in the reaction rates $k_{f}$ and $k_{r}$ from zero to non-zero values in the general gate equations is structural - it changes the underlying feedback structure of the system and, in particular, the monotone character of the equations. The same perturbation in the NCR gate does not change the feedback structure of the system.

The same comments apply to perturbations from the closed gate $k_{r}=0$. With this in mind we pose the following question: 
Within the constraints of the model for the full gate, i.e. the hypotheses $(\mathbf{H})$ and sufficiently large $r$ with respect to $\tau$ is it possible that for $0<k_{f}<<1$ or $0<k_{r}<<1$, either the attractor consists of more than a unique periodic orbit of period $r$ or that there is a unique periodic orbit but its period differs from a multiple of $r$ ?

As was indicated in the Introduction part of our justification for developing the full gate model was the observation that compartmental gating appears to be a wide spread phenomenon and clearly will appear in biological settings which have not yet been explored. Never the less, we do not know of a specific biological system for which the general model applies. There are two possible directions of speculation at this point. First, such a general gate will be found - justifying the analysis presented here. The second, is that the general gate does not exist. Given the enormous variety of biological control mechanisms which have arisen through evolution, this requires an explanation. Assuming a positive response to the question posed above, we provide the following obviously speculative remark. As is indicated in the comments following Theorem 1.3, the fact that the asymptotic dynamics is periodic with the period of the cell cycle allows for the output of the gate to be easily incorporated into other control mechanisms that are tuned to the cell cycle. Thus, evolution of this type of a switch might be less disruptive than, a switch whose output is either not unique or a signal with frequencies which are not easily related to that of the cell cycle.

Acknowledgements: K.M. was partially supported by NSF DMS 0443827 and grants from DOE and DARPA. T. G. was partially supported by NSF/NIH grant 0443863 , NIH-NCRR grant PR16445, NSF-BITS grant 0129895 and NSF-CRCNS grant 0515290 . E.M.B was partially supported by NSF DMS 0443855.

We would like to thank Peter Poláčik for discussions that led to the perturbation results of this paper.

\section{References}

[1] R. Albert, H. Othmer, The topology of the regulatory interactions predicts the expression pattern of the segment polarity genes in Drosophila melanogaster,J. Theor. Biology 223, 1 (2003)

[2] P. Bates, K. Lu and Ch. Zheng, Existence and persistence of invariant manifolds for semi-flows in Banach Spaces, Memoirs of AMS vol. 135, No. 645 (1998).

[3] M. P. Belvin, Y. Yin, K. V. Anderson, Cactus protein degradation mediates drosophila dorsol-ventral signalling. (1995) Genes Dev. 9:783-793.

[4] A. Brunet and A. Bonni, Akt promotes cell survival by phosphorylating and inhibiting a forkhead transcription factor. (1999) Cell 96, 6, 857-868.

[5] W. R. Burack and A. S. Shaw, Live cell imaging of ERK and MEK. (2005) J. Biol. Chem. 280:3832-3837.

[6] Hoffman-Bang, J., Nitrogen catabolite repression in Saccharomyces cerevisiae, Mol. Biotechnol. 12, 35-73, (1999).

[7] T. Beck and M. N. Hall, The TOR signalling pathway controls nuclear localization of nutrient regulated transcription factors. (1999) it Nature 402:689-692.

[8] E. Boczko, T. G. Cooper, T. Gedeon, K. Mischaikow, D. Murdock, S. Pratap and S. Wells, Structure Theorems and the Dynamics of Nitrogen Catabolite Repression in Yeast, PNAS vol. 102, no.16 (2005) $5647-5652$.

[9] Cooper,T.G., Transmitting the signal of excess nitrogen in Saccharomyces cerevisiae from the Tor proteins to the GATA factors: connecting the dots, FEMS microbiology Reviews 26, No.3 ,223-238, (2002).

[10] S-N. Chow and K. Lu, Invariant manifolds for flows in Banach spaces, JDE 74, 285-317 (1988).

[11] J. Hale, Asymptotic behavior of dissipative systems, AMS Mathematical Surveys and Monographs 25, AMS, Providence R.I., 1988. 
[12] J. Hale and S. Verduyn-Lunel, Introduction to Functional Differential Equations, Applied Mathematical Sciences 99, Springer-Verlag 1993.

[13] M.W. Hirsch, C.C. Pugh and M. Shub, Invariant manifolds, Lecture Notes in Mathematics, vol. 583, Springer-Verlag, New York, 1977.

[14] Y.-T. Juang, E. E. Solomou, B. Rellahan and G. C. Tsokos, Phosphorylation and O-linked glycosylation of Elf-1 leads to its translocation to the nucleus and to the promoter of the TCR $\zeta$-chain. (2002) $J$. Immunology 168:2865-2871.

[15] P. Kogerman, T. Grimm, L. Kogerman, D. Krause, A. Undén, B. Sandstedt, R. Toftgard and P. G. Zaphiropoulos, Mammalian suppressor of fused modulates nuclear-cytoplasmic shuttling of GLI-1. (1999) Nat. Cell. Bio. 1, 5, 312-319.

[16] K. Kondoh, K. Terasawa, H. Morimoto, and E. Nishida, Regulation of nuclear translocation of extracellular signal-regulated kinase 5 by active nuclear import and export mechanisms. (2006) Mol. Cell. Bio. 26:1679-1690.

[17] Krein, M.G. and Rutman, M.A., Linear operators leaving invariant cone in a Banach space, Uspechi Mat. Nauk 3, 3-95, A.M.S. Translation No. 26. Amer. Math. Soc., Providence, R.I., (1950)

[18] Mahaffy,J.M., Variation in Concentrations of RNAs and Proteins Involved in Gene Expression of Escherichia coli, J. Theor. Biol. 162, 153-186, (1993).

[19] R. Milo, S. Shen-Orr, S. Itzkovitz, N. Kashtan, D. Chklovskii and U. Alon, Network Motifs: Simple Building Blocks of Complex Networks Science, 298:824-827 (2002).

[20] S. Shen-Orr, R. Milo, S. Mangan and U. Alon, Network motifs in the transcriptional regulation network of Escherichia coli. Nature Genetics, 31:64-68 (2002).

[21] G. von Dassow, E. Meir, E. M. Munro and G. M. Odell., The segment polarity network is a robust developmental module, Nature 406, p. 188 (2000).

[22] M. Fabro and B. R. Henderson, Regulation of tumor suppressors by nuclear-cytoplasmic shuttling. (2003) Experimental Cell Research 282:59-69.

[23] G. Peng and J. E. Hopper, Gene activation by interaction of an inhibitor with a cytoplasmic signalling protein. (2002) Proc. Natl. Acad. Sci. 99:8548-8553.

[24] Ribbeck,K. \& Gorlich,D. Kinetic analysis of translocation through nuclear pore complexes, (2001) EMBO 20, 1320-1330.

[25] G. Skretas and D. W. Wood, Regulation of protein activity with small molecule inteins. (2005) Protein Science 14:523-532.

[26] Smith,H.,Monotone Dynamical Systems, AMS Mathematical Surveys and Monographs 41, AMS, Providence R.I., 1995.

[27] T. L. Tootle and I. Rebay, Post translational modifications influence transcription factor activity: a view from the ETS superfamily. (2005) BioEssays 27:285-298.

[28] H. Vaidyanathan and J. W. Ramos, RSK2 activity is regulated by its interaction with PEA-15. (2003) J. Biol. Chem. 278:32367-32372.

[29] M. Vandromme, C. Gauthier-Rouviere, N. Lamb and A. Fernandez, Regulation of transcription factor localization: fine tuning of gene expression. (1996) Trends Biochem. Sci. 21:59-64.

[30] C.-Y. Wang, B. Petryniak, C. B. Thompson, W. G. Kaelin, J. M. Leiden, Regulation of the Ets-related transcription factor Elf-1 by binding to the retinoblastoma protein. (1993) Science 260:1330-1335. 
[31] A. J. Whitmarsh, and R. J. Davis, Regulation of transcription factor function by phosphorylation. (2000) Cell. Mol. Life Sci. 57:1172-1183.

[32] W. W. Winkler, A. Nahvi, N. Sudarsan, J. E. Barrick, and R. R. Breaker, An mRNA structure that controls gene expression by binding S-adenosylmethionine. (2003) Nat. Struct. Biol. 10:701-707.

[33] Zeidler, E., Nonlinear Functional Analysis and its Applications I. Fixed Point Theorems, SpringerVerlag, New York 1986.

[34] Zhao X-Q., Dynamical Systems in Population Biology, Springer-Verlag, New York, 2003.

[35] Zhao X-Q., Asymptotic behavior for asymptotically periodic semi-flows with applications, Comm. Applied Nonlinear Analysis 3, No.4, (1996). 43-66. 